\title{
GALOIS INVARIANCE OF LOCAL ROOT NUMBERS
}

\author{
DAVID E. ROHRLICH
}

It is a standard remark that the group $\operatorname{Aut}(\mathbb{C})$ of abstract field automorphisms of $\mathbb{C}$ has uncountably many elements but only two that are are continuous. One consequence is that the analytic properties of a Dirichlet series $\sum_{n \geqslant 1} a(n) n^{-s}$ do not usually carry over to $\sum_{n \geqslant 1} \iota(a(n)) n^{-s}$ for $\iota \in \operatorname{Aut}(\mathbb{C})$. Silly counterexamples abound: for instance, take $a(n)=(1-\sqrt{2})^{n}$ and choose $\iota$ so that $\iota(\sqrt{2})=-\sqrt{2}$; then $\sum_{n \geqslant 1} a(n) n^{-s}$ converges everywhere but $\sum_{n \geqslant 1} \iota(a(n)) n^{-s}$ nowhere.

On the other hand, suppose that $\sum_{n \geqslant 1} a(n) n^{-s}$ is the Dirichlet series $L(s, M)$ associated to a motive $M$ over a number field (and to our implicit complex embedding of the coefficient field of $M)$. In this setting one does expect the Dirichlet series $L(s, M, \iota)=\sum_{n \geqslant 1} \iota(a(n)) n^{-s}$ to share certain analytic properties with $L(s, M)$ $(=L(s, M, \mathrm{id}))$. A case in point is the conjecture of Deligne and Gross: If $M$ is pure of odd weight then the order of vanishing of $L(s, M, \iota)$ at the center of the critical strip is independent of $\iota$ ([3], p. 323, Conjecture 2.7, part (ii)). This conjecture has a number of elementary consequences; for example, it enables one to deduce a "Birch and Swinnerton-Dyer conjecture with twists" from the usual version (cf. [10], p. 127). In this note we examine the consequences for root numbers.

The connection with root numbers depends on one further hypothesis. We have already assumed that the weight $w$ of $M$ is odd and hence that the center of the critical strip $(w+1) / 2$ is an integer, but suppose that in addition, $M$ is essentially self-dual. In other words, suppose that $M^{\vee} \cong M(w)$, where $M^{\vee}$ and $M(w)$ are respectively the dual and the $w$-fold Tate twist of $M$. The conjectured functional equation of $L(s, M, \iota)$ is then a relation bewtween $L(s, M, \iota)$ and itself, whence the root number $W(M, \iota)$ satisfies

$$
W(M, \iota)=(-1)^{\operatorname{ord}_{s=(w+1) / 2} L(s, M, \iota)}
$$

and the Deligne-Gross conjecture implies that $W(M, \iota)$ is independent of $\iota$. Our goal is to derive this conclusion unconditionally.

In practice what we prove is a purely local statement. Indeed the global root number $W(M, \iota)$ is a product of local root numbers, one for each place of the number field over which $M$ is defined, and at the infinite places the local root number is completely determined by the Hodge numbers of $M$. Since these Hodge numbers are known to be independent of $\iota$ by a theorem of Deligne ([3], p. 323, Proposition $2.5)$, it suffices to see that the local root numbers at the finite places are likewise invariant. Here the local root numbers are associated to representations of the local Weil group or Weil-Deligne group, so the problem reduces to an invariance property of root numbers associated to local representations. It is the latter property which 
is actually the subject of this note, and in fact after this introduction we shall make no further reference to motives at all. There is one lingering issue: Does the class of local representations to be considered here include the representations coming from an arbitrary essentially self-dual motive $M$ of odd weight? The point is that we require our local representations to be not only "essentially self-dual of odd weight" but also even-dimensional and "of essentially trivial determinant." Since $w$ is odd, the even-dimensionality follows from the Hodge realization of $M$, but I do not know whether the essential triviality of the determinant is likewise automatic. Of course this condition does hold whenever the $\lambda$-adic representations associated to $M$ are "essentially symplectic," as in Gross [8], p. 532, (3.7). In particular, our results cover the case of motives associated to $H^{1}$ of an abelian variety.

Setting this question aside for the time being, we may describe the contents of the paper as follows. We begin in Section 1 by proving an analogue for symplectic representations of Serre's induction theorem [12] for orthogonal representations, and then in Section 2 we state our main result on the Galois invariance of local root numbers (Theorem 1). The proof is given in Sections 3 and 4 and consists of a sequence of reductions, the most important of which is the replacement of an arbitrary irreducible symplectic representation by a two-dimensional monomial one. This is where we use the result proved in Section 1. Finally, in Section 5 we observe that while our main result was initially stated for representations of the Weil group, it remains valid for representations of the Weil-Deligne group (Theorem 2).

It is a bit disturbing that this paper is as long as it is. After all, the underlying issue is the behavior under Galois automorphisms of some square roots of rational numbers; surely this can be dealt with in a few pages! The reader is encouraged to try. At the very least it should be possible to simplify the proof of Proposition 2. Our proof of Proposition 2 was suggested by an argument of Feit generalizing a result of L. Solomon (see [4], pp. $73-76$, (14.3)), and while the result of FeitSolomon does not give Proposition 2 as a formal consequence, it is in principle much deeper. It would not be surprising if the proof of this deeper result turned out to be an inappropriate model for the proof of Proposition 2.

It is a pleasure to thank Ralph Greenberg for asking me a question which was the stimulus for this work.

1. A symplectic analogue of Serre's induction theorem. Throughout this paper, group representations are understood to be finite-dimensional and defined over the complex numbers. Given a group $G$ and a representation $\lambda$ of $G$, we write $\operatorname{tr} \lambda$ and $\lambda^{\vee}$ for the character and dual of $\lambda$ respectively, and we let $[\lambda]$ denote the class of $\lambda$ in the Grothendieck group of virtual representations of $G$. If $H$ is a subgroup of finite index in $G$ then $\operatorname{ind}_{H}^{G}$ denotes induction from $H$ to $G$, either of characters or of representations, and $\operatorname{res}_{H}^{G}$ denotes restriction from $G$ to $H$. But often the restriction to $H$ of a character or representation $\xi$ of $G$ will be denoted simply $\xi \mid H$.

We need to recall some basic facts about orthogonal and symplectic representations, starting with the definition: A representation $\lambda$ of $G$ is orthogonal if its space $V$ admits a nondegenerate symmetric bilinear form invariant under $G$, and $\lambda$ is symplectic if $V$ admits a nondegenerate alternating bilinear form invariant under $G$. This much is valid for any group and any representation, but if $G$ is finite and $\lambda$ irreducible then there is also a characterization involving the rationality properties of the matrix coefficients and character values: $\lambda$ is orthogonal if and only if it is 
realizable over $\mathbb{R}$, and $\lambda$ is symplectic if and only if $\operatorname{tr} \lambda$ is real-valued but $\lambda$ is not realizable over $\mathbb{R}$. As the self-dual representations of $G$ are precisely those with real-valued character, it follows that if $\lambda$ is both irreducible and self-dual then $\lambda$ is either orthogonal or symplectic.

If we drop the irreducibility requirement and assume only that $G$ is finite then it is still true that a representation is orthogonal if and only if it is realizable over $\mathbb{R}$, but it is no longer true that a representation with real-valued character is symplectic if and only if it is not realizable over $\mathbb{R}$. Nonetheless in both cases there is a characterization in terms of direct summands: A representation $\lambda$ of $G$ is orthogonal if and only if it is a direct sum of irreducible orthogonal representations and representations of the form $\theta \oplus \theta^{\vee}$, where $\theta$ denotes an arbitrary representation of $G$ (but since we are forming direct sums we can require $\theta$ to be irreducible if we like). Similarly, $\lambda$ is symplectic if and only if it is a direct sum of irreducible symplectic representations and representations of the form $\theta \oplus \theta^{\vee}$. We have already mentioned that $\lambda$ is self-dual if and only if $\operatorname{tr} \lambda$ is real-valued, but here too there is a characterization in terms of direct summands: $\lambda$ is self-dual if and only if it is a direct sum of irreducible orthogonal representations, irreducible symplectic representations, and representations of the form $\theta \oplus \theta^{\vee}$. Alternatively, since any representation of the form $\theta \oplus \theta^{\vee}$ is both orthogonal and symplectic, we can say that $\lambda$ is self-dual if and only if it is the direct sum of an orthogonal representation and a symplectic representation.

Finally, we remark that both orthogonality and symplecticity are stable under $\operatorname{ind}_{H}^{G}$ and $\operatorname{res}_{H}^{G}$. In the case of $\operatorname{res}_{H}^{G}$ the existence of the required invariant forms is immediate, because the space of the representation is unchanged by restriction, and in the case of $\operatorname{ind}_{H}^{G}$ one can construct an appropriate invariant form on the induced space from the given invariant form on the space of the inducing representation. For finite $G$ the argument that $\operatorname{ind}_{H}^{G}$ preserves orthogonality is even easier, because $\operatorname{ind}_{H}^{G}$ clearly preserves realizability over $\mathbb{R}$. There is also an easy argument for finite $G$ that $\operatorname{ind}_{H}^{G}$ preserves symplecticity: Combine Frobenius reciprocity with the fact that a self-dual representation of $G$ is symplectic if and only if the multiplicity of every irreducible orthogonal subrepresentation is even.

The facts just reviewed will be used without reference in the pages that follow. Our goal in this section is the following:

Proposition 1. Let $G$ be a finite group and $\lambda$ a symplectic representation of $G$. Then $[\lambda]$ can be written as an integral linear combination of classes of the form $\left[\theta \oplus \theta^{\vee}\right]$, where $\theta$ denotes an arbitrary irreducible representation of $G$, together with classes of the form $\left[\operatorname{ind}_{J}^{G} \sigma\right]$, where $J$ denotes a subgroup of $G$ and $\sigma$ a twodimensional irreducible monomial symplectic representation of $J$.

Statements of this sort are typically proved using elementary subgroups or their generalizations. Here it is the most inclusive generalization that is needed: recall that a finite group $H$ is called hyperelementary (or quasi-elementary, or $\mathbb{Q}$ elementary) if it has the structure of a semidirect product $C \rtimes P$, where $P$ is a $p$-group for some prime $p$ and $C$ a cyclic group of order prime to $p$. Let us denote the trivial one-dimensional character of a group $X$ by $1_{X}$. According to a theorem of L. Solomon (see Goldschmidt and Isaacs [7], p. 192, Theorem 3) we can write

$$
1_{G}=\sum_{H} n_{H} \operatorname{ind}_{H}^{G} 1_{H},
$$


where $H$ runs over hyperelementary subgroups of $G$ and $n_{H} \in \mathbb{Z}$ for all $H$. Multiplying both sides of (1.1) by $\operatorname{tr} \lambda$, we obtain

$$
\operatorname{tr} \lambda=\sum_{H} n_{H} \operatorname{tr}\left(\operatorname{ind}_{H}^{G}\left(\operatorname{res}_{H}^{G} \lambda\right)\right)
$$

or in other words

$$
[\lambda]=\sum_{H} n_{H}\left[\operatorname{ind}_{H}^{G}\left(\operatorname{res}_{H}^{G} \lambda\right)\right]
$$

Now each $\operatorname{res}_{H}^{G} \lambda$ in (1.2) is again symplectic, hence a direct sum of (i) irreducible symplectic representations $\tau$ of $H$ and (ii) representations of the form $\zeta \oplus \zeta^{\vee}$, where $\zeta$ is an arbitrary irreducible representation of $H$. Given a representation of type (ii), we can write $\operatorname{ind}_{H}^{G} \zeta$ as a direct sum of irreducible representations $\theta$, and then $\operatorname{ind}_{H}^{G}\left(\zeta \oplus \zeta^{\vee}\right)$ is the corresponding direct sum of the representations $\theta \oplus \theta^{\vee}$. As for representations of type (i), the following proposition enables us to write $\operatorname{ind}_{H}^{G} \tau=\operatorname{ind}_{J}^{G} \sigma$ with $\sigma$ as in Proposition 1.

Proposition 2. Let $H$ be a hyperelementary group and $\tau$ an irreducible symplectic representation of $H$. Then there is a subgroup $J$ of $H$ and a two-dimensional symplectic representation $\sigma$ of $J$ such that $\tau=\operatorname{ind}_{J}^{H} \sigma$.

We have not stated explicitly that $\sigma$ is irreducible monomial because this is an immediate consequence: $\sigma$ induces the irreducible representation $\tau$ and is therefore irreducible, and $J$ is a subgroup of a hyperelementary group, hence itself hyperelementary, hence supersolvable, and consequently every irreducible representation of $J$ is monomial. We conclude that Proposition 1 is a consequence of Proposition 2 and that the subgroups $J$ in Proposition 1 can be taken to be hyperelementary.

The proof of Proposition 2 will occupy several paragraphs. We begin with a slight refinement of the fact that irreducible representations of $H$ are monomial:

Lemma. Write $H=C \rtimes P$, where $P$ is a $p$-group for some prime $p$ and $C$ a cyclic group of order prime to $p$. Then every irreducible representation of $H$ is induced from a one-dimensional representation of a subgroup of $H$ containing $C$.

Proof. This is a standard remark if the words "containing $C$ " are omitted (cf. [15], p. 61, Prop. 24) and a routine exercise in any case. Nevertheless, we briefly sketch the argument. Let $\tau$ be an irreducible representation of $H$ and $V$ the space of $\tau$. We consider cases according as $\tau \mid C$ is scalar or nonscalar.

If $\tau \mid C$ is scalar then $\tau \mid P$ is irreducible, and since $p$-groups are supersolvable, $\tau \mid P$ is monomial. Hence there is a subgroup $Q$ of $P$ and a $Q$-stable line $W$ in $V$ such that $V$ is the direct sum of the lines $\tau(x) W$, where $x$ runs over a set of representatives for the distinct left cosets of $Q$ in $P$. But as $\tau \mid C$ is scalar, $W$ is stable under the larger subgroup $C \rtimes Q$, and since the elements $x$ also represent the distinct left cosets of $C \rtimes Q$ in $H$ we conclude that $\tau$ is induced by a one-dimensional representation of $C \rtimes Q$.

Next suppose that $\tau \mid C$ is nonscalar. Since $C$ is abelian but $\tau \mid C$ nonscalar there is a one-dimensional character $\chi$ of $C$ such that the $\chi$-isotypic component of $V$, say $W$, satisfies $\{0\} \varsubsetneqq W \varsubsetneqq V$. Let $Q$ be the subgroup of $P$ consisting of elements $g \in P$ such that $\chi\left(g c g^{-1}\right)=\chi(c)$ for $c \in C$. Then $W$ is stable under $C \rtimes Q$ 
and $\tau$ is induced by the representation - call it $\pi$ - of $C \rtimes Q$ on $W$. Now $\pi$ is irreducible because $\tau$ is, and $\pi \mid C$ is scalar because $W$ is $\chi$-isotypic. Hence by the case of the lemma already proved, $\pi$ is induced by a one-dimensional representation of some subgroup of $C \rtimes Q$ containing $C$. Thus the same is true of $\tau$. We remark that instead of appealing to the case of the lemma already proved, we could have completed the argument by induction on $|H|$ : indeed $Q$ is a proper subgroup of $P$ because $W$ is $Q$-stable whereas $\tau$ is irreducible.

Proof of Proposition 2. As noted in the introduction, the idea of the proof is derived from Feit [4], pp. $73-76,(14.3)$. But since the connection with the latter argument may not be obvious, we shall provide full details, incorporating only those elements of [4] which are relevant to our situation.

Write $H=C \rtimes P$ as in the lemma. Then any subgroup of $H$ containing $C$ has the form $C \rtimes Q$ with a subgroup $Q$ of $P$. We shall write $C \rtimes Q$ simply as $C Q$, and for the sake of congruity we shall often write $H$ as $C P$. By the lemma, there is a subgroup $Q$ of $P$ and a one-dimensional representation $\xi$ of $C Q$ such that $\tau \cong \operatorname{ind}_{C Q}^{C P} \xi$. It follows that $\operatorname{dim} \tau=[P: Q]=p^{n}$ for some integer $n \geqslant 0$. But $\tau$ is symplectic, hence even-dimensional. Consequently $p=2$ and $Q$ is a proper subgroup of $P$. In particular there is a subgroup $R$ of index 2 in $P$ which contains $Q$, and we can write $\tau=\operatorname{ind}_{C R}^{C P} \theta$ with $\theta=\operatorname{ind}_{C Q}^{C R} \xi$. Note that $\theta$ is irreducible since it induces the irreducible representation $\tau$. Furthermore $|C R|=|H| / 2$, so if $\theta$ is symplectic then by induction on $|H|$ there is a subgroup $J$ of $C R$ and a two-dimensional symplectic representation $\sigma$ of $J$ such that $\theta=\operatorname{ind}_{J}^{C R} \sigma$. Then $\tau=\operatorname{ind}_{J}^{H} \sigma$ and we are done. So we may assume that $\theta$ is not symplectic. On the other hand, $\theta$ is not orthogonal, else the representation $\tau=\operatorname{ind}_{C R}^{C P} \theta$ is also orthogonal, contradicting the fact that $\tau$ is symplectic. As $\theta$ is irreducible but neither symplectic nor orthogonal we conclude that $\theta$ is not self-dual.

To summarize, $\tau=\operatorname{ind}_{C R}^{C P} \theta$ with $[P: R]=2$ and $\theta \not \theta^{\vee}$. If $\operatorname{dim} \theta=1$ then we are done: the desired formula $\tau=\operatorname{ind}_{J}^{H} \sigma$ holds with $J=H$ and $\sigma=\tau$, because $\sigma$ is then a two-dimensional symplectic representation of $J$. So we may assume that $\operatorname{dim} \theta>1$. Now by construction (or if we have forgotten the construction then by another appeal to the lemma) there is a subgroup $Q$ of $R$ and a one-dimensional representation $\xi$ of $C Q$ such that $\theta=\operatorname{ind}_{C Q}^{C R} \xi$. If $Q=R$ then $\operatorname{dim} \theta=1$, a contradiction. Hence $Q$ is a proper subgroup of the 2-group $R$, and consequently there is a subgroup $S$ of index 2 in $R$ which contains $Q$. And since $\theta$ is induced from $C Q$ it is also induced from $C S$.

Now comes the crucial step in the proof: We claim that $\theta$ is induced from $C T$ for some subgroup $T$ of index 2 in $R$ which is normal in $P$. If $S$ is itself normal in $P$ then we can take $T=S$, so in verifying the claim we shall assume that $S$ is not normal. Since $[P: S]=[P: R][R: S]=4$, it is natural to write $\mathcal{S}_{4}$ for the group of permutations of the coset space $P / S$. Then the action of $P$ on $P / S$ by left multiplication gives a homomorphism $f: P \rightarrow \mathcal{S}_{4}$. Now the kernel of $f$ is the intersection of the conjugates of $S$ in $P$, and this intersection is a proper subroup of $S$ because $S$ is not normal. Since $|f(P)|=[P: S][S: \operatorname{ker} f]=4[S: \operatorname{ker} f]$ it follows that $|f(P)|$ is divisible by 8. But a Sylow 2-subgroup of $\mathcal{S}_{4}$ is isomorphic to $D_{8}$ (the dihedral group of order 8 ) so we conclude that $f(P) \cong D_{8}$ and that $[S: \operatorname{ker} f]=2$. Let $T$ be the inverse image under $f$ of the center of $f(P)$. Then $T$ is a normal subgroup of $P$ of index 4 , and $T$ is contained in $R$ (necessarily with index 2) because the center of $D_{8}$ is contained in every subgroup of order 4 . (In 
other words, the calculation $|f(R)|=[R: \operatorname{ker} f]=[R: S][S: \operatorname{ker} f]=4$ allows us to conclude that $f(T) \subset f(R)$.) To complete the proof of the claim we must show that $\theta$ is induced from $C T$.

Before doing so, we make two remarks. The first pertains to ker $f$. We have already noted that $\operatorname{ker} f$ is the intersection of the conjugates of $S$ in $P$, and the remark is that since $S$ is normal in $R$ (for $[R: S]=2$ ) there are just two conjugates: $S$ itself and $g S g^{-1}$, where $g$ is any representative of the nontrivial coset of $R$ in $P$. The second remark is that for such $g$ we have $\theta_{g} \cong \theta^{\vee}$, where $\theta_{g}$ is the representation $x \mapsto \theta\left(g^{-1} x g\right)$ of $C R$. Indeed since $\tau=\operatorname{ind}_{C R}^{C P} \theta$ we have $\operatorname{res}_{C R}^{C P} \tau=\theta \oplus \theta_{g}$, but $\tau$ is self-dual whereas $\theta$ is not, so $\operatorname{res}_{C R}^{C P} \tau=\theta \oplus \theta^{\vee}$.

Let us now prove that $\theta$ is induced from $C T$. We know that $\theta$ is induced from $C S$, and since $C S$ is normal in $C R$ the formula for an induced character shows that $\operatorname{tr} \theta$ vanishes on the complement $C R \backslash C S$. It follows that $\operatorname{tr} \theta_{g}$ vanishes on $C R \backslash C g S g^{-1}$. But we have just seen that if $g \in P \backslash R$ then $\operatorname{tr} \theta_{g}=\overline{\operatorname{tr} \theta}$, so we conclude that $\operatorname{tr} \theta$ itself vanishes on $C R \backslash C g S g^{-1}$ as well as on $C R \backslash C S$. So $\operatorname{tr} \theta$ vanishes on $C R \backslash \operatorname{ker} f$ and hence a fortiori on $C R \backslash C T$. Since $[C R: C T]=2$ it follows that $\theta$ is induced from $C T$.

Having proved the claim, we write $\theta=\operatorname{ind}_{C T}^{C R} \mu$ with $\mu$ a representation of $C T$. Next we appeal to a general fact: if $G$ is a finite group and $N$ a normal subgroup then two representations of $N$ which induce the same irreducible representation of $G$ are conjugate under $G$. In fact if a representation $\kappa$ of $N$ induces a given irreducible representation $\pi$ of $G$ then the map $g N \mapsto\left[\kappa_{g}\right]$ is a bijection from $G / N$ to the set of isomorphism classes of representations of $N$ inducing $\pi$. In the case at hand we may take $G=H=C P, N=C T, \pi=\tau$, and $\kappa=\mu$; indeed $\operatorname{ind}_{C T}^{C P} \mu=\operatorname{ind}_{C R}^{C P} \theta=\tau$. But $\tau$ is self-dual, so $\mu^{\vee}$ also induces $\tau$, and consequently there is an element $h \in H$ such that $\mu_{h} \cong \mu^{\vee}$. Note that $h \notin C T$, else $\mu$ is self-dual, contradicting the fact that $\mu$ induces $\theta$. But the fact that $\operatorname{tr} \mu_{h}=\overline{\operatorname{tr} \mu}$ implies that $\mu_{h^{2}} \cong \mu$ and hence that $h^{2} \in C T$. This last fact combined with the normality of $C T$ ensures that the set $K=(C T) \cup h(C T)$ is a subgroup of $H$. Furthermore $\tau=\operatorname{ind}_{K}^{H} \nu$, where $\nu=\operatorname{ind}_{C T}^{K} \mu$. As $\mu$ is conjugate to $\mu^{\vee}$ via the nontrivial coset of $C T$ in $K$, we see that the irreducible representation $\nu$ is self-dual, hence either orthogonal or symplectic. If $\nu$ is orthogonal then so is $\tau$, a contradiction. So $\nu$ is symplectic, and since $|K|=|H| / 2$ we may assume by induction that there is a subgroup $J$ of $K$ and a two-dimensional symplectic representation $\sigma$ of $J$ such that $\nu=\operatorname{ind}_{J}^{K} \sigma$. Then $\tau=\operatorname{ind}_{J}^{H} \sigma$.

While the proof of Proposition 1 is now complete, we also need an ancillary remark concerning the underlying one-dimensional characters. First we introduce some additional notation.

Given a finite group $J$ and a subgroup $I$, let $\operatorname{trans}_{I}^{J}: J^{\mathrm{ab}} \rightarrow I^{\mathrm{ab}}$ denote the transfer, where the superscript "ab" on a finite group indicates the maximal abelian quotient. If $\chi$ is a one-dimensional character of $I$ then $\chi$ factors through $I^{\mathrm{ab}}$, and thus we can form the composition $\chi \circ \operatorname{trans}_{I}^{J}$, which we then view as a onedimensional character of $J$. Another one-dimensional character of $J$ appearing in the following proposition is the sign of the permutation representation of $J$ on the left cosets of $I$, which we denote $\operatorname{sign}_{J / I}$.

Proposition 3. Let $J$ be a finite group and $\sigma$ a two-dimensional irreducible monomial self-dual representation of $J$. Then there is a subgroup $I$ of index two in $J$ 
and a one-dimensional character $\chi$ of $I$ such that $\sigma=\operatorname{ind}_{I}^{J} \chi$ and either

(a) $\chi \circ \operatorname{trans}_{I}^{J}=1_{J}$, or

(b) $\chi \circ \operatorname{trans}_{I}^{J}=\operatorname{sign}_{J / I}$

according as $\sigma$ is orthogonal or symplectic.

Proof. This is standard, but we nonetheless run through the details, splitting the proof into cases according as $\sigma$ is (a) orthogonal or (b) symplectic. The formula

$$
\operatorname{det} \sigma=\left(\operatorname{sign}_{J / I}\right)\left(\chi \circ \operatorname{trans}_{I}^{J}\right)
$$

(cf. [6] or [1], p. 508, Proposition 1.2) will be used in both cases.

(a) If $\sigma$ is orthogonal then it can be viewed as a homorphism $J \rightarrow \mathrm{O}(2, \mathbb{R})$, but its image is not contained in $\mathrm{SO}(2, \mathbb{R})$ because $\mathrm{SO}(2, \mathbb{R})$ is abelian whereas $\sigma$ is irreducible. As $\mathrm{SO}(2, \mathbb{R})$ has index two in $\mathrm{O}(2, \mathbb{R})$ it follows that the subgroup $I=\sigma^{-1}(\mathrm{SO}(2, \mathbb{R}))$ has index two in $J$, and since $\sigma$ is irreducible while $I$ is abelian of index two we obtain $\sigma=\operatorname{ind}_{J}^{I} \chi$ for some character $\chi$ of $I$. Furthermore, the fact that $\sigma(I) \subset \mathrm{SO}(2, \mathbb{R})$ but $\sigma(J) \not \subset \mathrm{SO}(2, \mathbb{R})$ means that $\operatorname{det} \sigma$ is trivial on $I$ but not on $J$, whence $\operatorname{det} \sigma=\operatorname{sign}_{J / I}$. Consequently $\chi \circ \operatorname{trans}_{I}^{J}=1_{J}$ by (1.3). Note that the assumption that $\sigma$ is monomial is not needed in case (a).

(b) Next suppose that $\sigma$ is symplectic. Since $\sigma$ is by hypothesis also twodimensional and monomial, there is a subgroup $I$ of index two in $J$ and a character $\chi$ of $I$ such that $\sigma=\operatorname{ind}_{I}^{J} \chi$. The formula $\chi \operatorname{otrans}_{I}^{J}=\operatorname{sign}_{J / I}$ now follows from (1.3), because $\operatorname{Sp}(2)=\mathrm{SL}(2)$ : in other words, a nonsingular $2 \times 2$ matrix is symplectic if and only if its determinant is 1 . We remark that this time it is the irreducibility of $\sigma$ which is not used in the proof. Furthermore the formula $\chi \circ \operatorname{trans}_{I}^{J}=\operatorname{sign}_{J / I}$ holds for any $I$ and $\chi$ such that $\sigma=\operatorname{ind}_{I}^{J} \chi$.

2. Statement of the main theorem. Fix a prime number $p$. All finite extensions of $\mathbb{Q}_{p}$ appearing in this paper are assumed to lie inside some fixed algebraic closure of $\mathbb{Q}_{p}$. We denote this algebraic closure $\bar{F}$, because $F$ will serve as our default notation for a finite extension of $\mathbb{Q}_{p}$. We write $\mathcal{O}$ for the ring of integers of $F$ and $\mathfrak{p}$ for the maximal ideal of $\mathcal{O}$, and we put $q=|\mathcal{O} / \mathfrak{p}|$, so that $q=p^{f}$, where $f$ is the residue class degree. If we want to emphasize the dependence of these items on $F$, or if we are dealing with a finite extension of $\mathbb{Q}_{p}$ denoted by some letter other than $F$, then we append a subscript: $\mathcal{O}_{X}, \mathfrak{p}_{X}, q_{X}, f_{X}$ if the field in question is $X$.

We shall be concerned with representations of the Weil group $\mathrm{W}(\bar{F} / F)$, which like $\operatorname{Gal}(\bar{F} / F)$ is to be regarded here as a topological group. Thus a representation of $\mathrm{W}(\bar{F} / F)$ is understood to be a continuous homomorphism $\rho: \mathrm{W}(\bar{F} / F) \rightarrow$ $\mathrm{GL}(V)$, where $V$ is a finite-dimensional vector space over $\mathbb{C}$. In particular, a onedimensional character is a continuous homomorphism $\mathrm{W}(\bar{F} / F) \rightarrow \mathbb{C}^{\times}$.

Henceforth we shall refer to a one-dimensional character simply as a "character" when there is no possibility of confusion. For example, we can speak of the primeto- $p$ cyclotomic character $\omega$, which is the unramified character of $\mathrm{W}(\bar{F} / F)$ taking the value $q$ on Frobenius elements. Let $F^{\text {ab }}$ denote the abelian closure of $F$ inside $\bar{F}$, and write $\mathrm{W}(\bar{F} / F)^{\text {ab }}$ for the quotient of $\mathrm{W}(\bar{F} / F)$ by the closure of its commutator subgroup. Using the Artin map $x \mapsto\left(x, F^{\mathrm{ab}} / F\right)$ to identify $F^{\times}$with $\mathrm{W}(\bar{F} / F)^{\mathrm{ab}}$, we may view $\omega$ as a character of $F^{\times}$, but for the sake of consistency with [1] we shall agree that a character $\chi$ of $\mathrm{W}(\bar{F} / F)$ is made into a character of $F^{\times}$by setting

$$
\chi(x)=\chi\left(\left(x^{-1}, F^{\mathrm{ab}} / F\right)\right)
$$


for $x \in F^{\times}$. Thus the value of $\omega$ on a uniformizer is $q^{-1}$.

In addition to $\omega$ we shall encounter characters of the form $\omega^{w / 2}$ and $\left(\omega^{w / 2}\right)^{\iota}$, where $w \in \mathbb{Z}$ and $\iota \in \operatorname{Aut}(\mathbb{C})$. Quite generally, given a representation $\rho$ of $\mathrm{W}(\bar{F} / F)$, we define a representation $\rho^{\iota}$ of $\mathrm{W}(\bar{F} / F)$ up to isomorphism as follows: After choosing a basis for the space of $\rho$ we may view $\rho$ as a matrix representation $\rho: \mathrm{W}(\bar{F} / F) \rightarrow \mathrm{GL}(n, \mathbb{C}) ;$ then $\rho^{\iota}: \mathrm{W}(\bar{F} / F) \rightarrow \mathrm{GL}(n, \mathbb{C})$ is the representation obtained by applying $\iota$ to the matrix coefficients of $\rho(g)$ for $g \in \mathrm{W}(\bar{F} / F)$. In spite of the fact that $\iota$ is only an abstract field automorphism, $\rho^{\iota}$ is still continuous, as required, because the continuity of a homomorphism $\rho: \mathrm{W}(\bar{F} / F) \rightarrow \mathrm{GL}(n, \mathbb{C})$ is equivalent to the triviality of $\rho$ on an open subgroup of the inertia subgroup of $\mathrm{W}(\bar{F} / F)$. Returning to the case $\rho=\omega^{w / 2}$, we see that

$$
\left(\omega^{w / 2}\right)^{\iota}= \begin{cases}\omega^{w / 2} & \text { if } \iota(\sqrt{p})=\sqrt{p} \\ \eta^{w f} \cdot \omega^{w / 2} & \text { if } \iota(\sqrt{p})=-\sqrt{p}\end{cases}
$$

with $\eta$ the unramified quadratic character of $F^{\times}$.

Our main theorem will assert that for a certain class of representations $\rho$ of $\mathrm{W}(\bar{F} / F)$, the root number $W(\rho)$ is unchanged when $\rho$ is replaced by $\rho^{\iota}$. However in the first instance we should write $W(\rho, \psi)$ rather than $W(\rho)$, because the root number depends in general on the choice of an additive character of $F$, by which we mean a nontrivial unitary character $\psi: F \rightarrow \mathbb{C}$. Given such a $\psi$, we set

$$
W(\rho, \psi)=\frac{\epsilon(\rho, \psi, d x)}{|\epsilon(\rho, \psi, d x)|},
$$

where $d x$ is any Haar measure on $F$ and $\epsilon(\rho, \psi, d x)$ is defined as in [17], p. 14, (3.4.1). That said, we shall now focus on a class of representations $\rho$ for which $W(\rho, \psi)$ is in fact independent of $\psi$. We note in passing that in conjunction with a standard formula (cf. [17], p. 15, (3.4.6)), the definition (2.3) gives

$$
W\left(\rho \otimes \omega^{r}, \psi\right)=W(\rho, \psi)
$$

for any $r \in \mathbb{R}$, because $\omega^{r}$ is unramified and takes positive real values.

Let us call a representation $\rho$ of $\mathrm{W}(\bar{F} / F)$ essentially self-dual of weight $w$ if $\rho \otimes \omega^{w / 2}$ is self-dual, or equivalently if $\rho^{\vee} \cong \rho \otimes \omega^{w}$. By taking determinants of both sides, we see that for such a representation we have $(\operatorname{det} \rho)^{2}=\omega^{-w \operatorname{dim} \rho}$. We will say that the determinant of $\rho$ is essentially trivial if $\operatorname{det} \rho=\omega^{-(w \operatorname{dim} \rho) / 2}$. Thus $\operatorname{det} \rho$ is essentially trivial if $\operatorname{det}\left(\rho \otimes \omega^{w / 2}\right)$ is trivial.

Proposition 4. Let $\rho$ be a representation of $\mathrm{W}(\bar{F} / F)$.

(i) If $\rho$ is essentially self-dual and $\operatorname{det} \rho$ is essentially trivial then $W(\rho, \psi)$ is equal to \pm 1 and independent of $\psi$.

(ii) If $\rho$ is essentially self-dual then so is $\rho^{\iota}$.

(iii) Suppose that $\rho$ is essentially self-dual and $\operatorname{dim} \rho$ is even. If $\operatorname{det} \rho$ is essentially trivial then so is $\operatorname{det} \rho^{\iota}$.

(iv) If $\rho$ is essentially self-dual, even-dimensional, and of essentially trivial determinant then $W\left(\rho^{\iota}, \psi\right)$ is equal to \pm 1 and independent of $\psi$.

Proof. The proposition is a straightforward consequence of standard formulas, and our "proof" will consist largely of references to the literature. 
(i) Let $w$ be the weight of $\rho$. To see that $W(\rho, \psi)= \pm 1$, we start with the formula

$$
W(\rho, \psi) W\left(\rho^{\vee}, \psi\right)=\operatorname{det} \rho(-1)
$$

(cf. [17], p. 15, (3.4.7) and (3.4.4) - note that $\operatorname{det} \rho$ is identified here with a character of $F^{\times}$via (2.1), and in applying formula (3.4.7) of [17], use (2.4) above). As $\rho$ is essentially self-dual, we have

$$
W\left(\rho^{\vee}, \psi\right)=W\left(\rho \otimes \omega^{w}, \psi\right)=W(\rho, \psi),
$$

the second equality being another instance of (2.4). Since $\operatorname{det} \rho$ is essentially trivial and thus in particular unramified we have $\operatorname{det} \rho(-1)=1$, so that $(2.5)$ and $(2.6)$ give $W(\rho, \psi)^{2}=1$, as claimed.

To see that $W(\rho, \psi)$ is independent of $\psi$ we recall that if a particular additive character $\psi$ of $F$ is given, then every additive character of $F$ has the form $\psi_{c}$ for some $c \in F^{\times}$, where $\psi_{c}: F \rightarrow \mathbb{C}$ is the additive character $\psi_{c}(x)=\psi(c x)$. On the other hand, we have

$$
W\left(\rho, \psi_{c}\right)=\frac{(\operatorname{det} \rho)(c)}{|(\operatorname{det} \rho)(c)|} W(\rho, \psi)
$$

(cf. [17], p. 15, (3.4.4)), where $\operatorname{det} \rho$ is once again viewed as a character of $F^{\times}$using (2.1). Since $\operatorname{det} \rho$ coincides with $\omega^{-(w \operatorname{dim} \rho) / 2}$ and in particular takes values in the positive real numbers, the claimed independence follows from (2.7).

(ii) This is straightforward: $\left(\rho^{\iota}\right)^{\vee} \cong\left(\rho^{\vee}\right)^{\iota} \cong\left(\rho \otimes \omega^{w}\right)^{\iota} \cong \rho^{\iota} \otimes \omega^{w}$.

(iii) Also straightforward: $\operatorname{det} \rho^{\iota}=(\operatorname{det} \rho)^{\iota}=\left(\omega^{-(w \operatorname{dim} \rho) / 2}\right)^{\iota}=\omega^{-(w \operatorname{dim} \rho) / 2}$ because $\operatorname{dim} \rho$ is even.

(iv) This follows from (i), (ii), and (iii).

Henceforth, if $\rho$ is essentially self-dual and $\operatorname{det} \rho$ is essentially trivial then we write $W(\rho, \psi)$ simply as $W(\rho)$.

Theorem 1. Suppose that $\rho$ is essentially self-dual of odd weight, even dimension, and essentially trivial determinant. Then $W\left(\rho^{\iota}\right)=W(\rho)$.

The proof of Theorem 1 will be given in Sections 3 and 4. The remainder of the present section is devoted to a brief discussion of notation and formulas followed by three examples demonstrating the necessity of the hypotheses in Theorem 1. First the notation and formulas: If $\rho$ is a representation of $\mathrm{W}(\bar{F} / F)$ then $a(\rho)$ is the nonnegative integer such that $\mathfrak{p}^{a(\rho)}$ is the conductor of $\rho$, and if $\psi$ is an additive character of $F$ then $n(\psi)$ is the largest integer $n$ such that $\psi$ is trivial on $\mathfrak{p}^{-n}$. Since $\eta$ is the unramified quadratic character of $\mathrm{W}(\bar{F} / F)$, standard formulas give

$$
W(\rho \otimes \eta, \psi)=(-1)^{a(\rho)+n(\psi) \operatorname{dim} \rho} W(\rho, \psi)
$$

(cf. [17], p. 15, (3.4.6)) as well as

$$
W(\eta, \psi)=(-1)^{n(\psi)}
$$

(cf. [17], p. 13, (3.2.6.1)), and the latter citation also gives

$$
W\left(\omega^{r}, \psi\right)=1
$$


for any $r \in \mathbb{R}$. Thus (2.9) is the special case of (2.8) in which $\rho=\omega^{0}$.

We also need some notation pertaining to finite extensions $E / F$. In particular, $f(E / F)$ is the degree of the residue class field extension and $d(E / F)$ the integer such that $\mathfrak{p}^{d(E / F)}$ is the relative discriminant of $E / F$. Furthermore, if $E / F$ is quadratic then we write $\operatorname{sign}_{E / F}$ for the quadratic character of $\mathrm{W}(\bar{F} / F)$ trivial on $\mathrm{W}(\bar{F} / E)$. Note that in the quadratic case $d(E / F)$ has an alternative description as the integer such that $\mathfrak{p}^{d(E / F)}$ is the conductor of $\operatorname{sign}_{E / F}$. In other words, $a\left(\operatorname{sign}_{E / F}\right)=d(E / F)$. Returning to the case of a general finite extension $E / F$, let us agree that $\operatorname{ind}_{E / F}$ denotes induction either from $\operatorname{Gal}(\bar{F} / E)$ to $\operatorname{Gal}(\bar{F} / F)$ or from $\mathrm{W}(\bar{F} / E)$ to $\mathrm{W}(\bar{F} / F)$, depending on context. We write $N_{E / F}$ for the norm from $E$ to $F$.

Example 1. Take $f$ and $w$ to be odd and put $\rho=\omega^{-w / 2}$. If $\iota \in \operatorname{Aut}(\mathbb{C})$ restricts nontrivially to $\mathbb{Q}(\sqrt{p})$ then $(2.2)$ gives $\rho^{\iota}=\eta \cdot \omega^{w / 2}$, whence

$$
W\left(\rho^{\iota}, \psi\right)=(-1)^{n(\psi)}
$$

by (2.4) and (2.9). But $W(\rho, \psi)=1$ by $(2.10)$. Hence if we choose $\psi$ so that $n(\psi)$ is odd then $W\left(\rho^{\iota}, \psi\right)=-W(\rho, \psi)$, even though $\rho$ is essentially self-dual of odd weight and essentially trivial determinant. Thus the requirement that $\operatorname{dim} \rho$ be even cannot be omitted in Theorem 1.

Example 2. As in Example 1, we take $f$ and $w$ to be odd. Fix a uniformizer $\pi$ of $\mathcal{O}$ and a square root $\sqrt{\pi}$, put $E=F(\sqrt{\pi})$, and let $\rho=\left(1 \oplus \operatorname{sign}_{E / F}\right) \otimes \omega^{-w / 2}$. If $\iota(\sqrt{p})=-\sqrt{p}$ then $\rho^{\iota}=\rho \otimes \eta$ by $(2.2)$, whence

$$
W\left(\rho^{\iota}, \psi\right)=(-1)^{d(E / F)} W(\rho, \psi)
$$

by (2.8). Here we are using $\operatorname{dim} \rho=2$ and $a(\rho)=a\left(\operatorname{sign}_{E / F}\right)=d(E / F)$. But $\mathfrak{p}^{d(E / F)}$ is the relative discriminant of $E / F$, which is the ideal of $\mathcal{O}$ generated by $N_{E / F}\left(P^{\prime}(\sqrt{\pi})\right)$ for $P(x)=x^{2}-\pi$. Thus the relative discriminant of $E / F$ is $\pi \mathcal{O}$ or $4 \pi \mathcal{O}$ according as $p$ is odd or $p=2$, and in either case we conclude that $d(E / F)$ is odd. Returning to (2.11), we see that $W\left(\rho^{\iota}, \psi\right)=-W(\rho, \psi)$, in spite of the fact that $\rho$ is even-dimensional and essentially self-dual of odd weight. Hence the essential triviality of $\operatorname{det} \rho$ is a necessary hypothesis in Theorem 1 .

Example 3. We assume once again that $f$ is odd, but this time we take $w$ to be even. Furthermore, we fix an even-dimensional self-dual representation $\lambda$ of $\operatorname{Gal}(\bar{F} / F)$ with $\operatorname{det} \lambda$ trivial and $a(\lambda)$ odd. The existence of such a representation will be verified in the addendum below. (Note also that a representation of this type was exhibited by Serre in connection with his work on the rationality of Artin representations; see [13], pp. 412-414 and [14], pp. 180-181.) Viewing $\lambda$ as a representation of $\mathrm{W}(\bar{F} / F)$, we put $\rho=\lambda \otimes \omega^{-w / 2}$ and $\rho_{0}=\rho \otimes \omega^{-1 / 2}$, so that $\rho_{0}$ is essentially self-dual of odd weight, even dimension, and essentially trivial determinant. Thus $W\left(\rho_{0}^{\iota}\right)=W\left(\rho_{0}\right)$ by Theorem 1 . On the other hand, the relation $\rho_{0}=\rho \otimes \omega^{-1 / 2}$ gives $W\left(\rho_{0}\right)=W(\rho)$ by (2.4). Similarly, if $\iota(\sqrt{p})=-\sqrt{p}$ then $\rho_{0}^{\iota}=\rho^{\iota} \otimes\left(\eta \omega^{-1 / 2}\right)$ by $(2.2)$, whence (2.4) gives $W\left(\rho_{0}^{\iota}\right)=W\left(\rho^{\iota} \otimes \eta\right)$. So the equality $W\left(\rho_{0}^{\iota}\right)=W\left(\rho_{0}\right)$ becomes

$$
W\left(\rho^{\iota} \otimes \eta\right)=W(\rho)
$$


Since $a\left(\rho^{\iota}\right)=a(\rho)=a(\lambda)$, the left-hand side of $(2.12)$ is $(-1)^{a(\lambda)} W\left(\rho^{\iota}\right)$ by $(2.8)$. But $a(\lambda)$ is odd, so we conclude that $W\left(\rho^{\iota}\right)=-W(\rho)$, even though $\rho$ is essentially self-dual of even dimension and essentially trivial determinant. Thus the assumption that $w$ is odd is necessary in Theorem 1 .

Addendum to Example 3. We must still verify that a representation $\lambda$ as above exists. As in Example 2, we put $E=F(\sqrt{\pi})$, but in the first instance we view $\operatorname{sign}_{E / F}$ as a character of $F^{\times}$. Let $x \mapsto x^{\prime}$ be the nontrivial element of $\operatorname{Gal}(E / F)$. We shall construct a character $\chi$ of $E^{\times}$of finite order satisfying the following conditions:

(i) $\chi \mid F^{\times}=\operatorname{sign}_{E / F}$.

(ii) $\chi\left(x^{\prime}\right)=\chi(x)^{-1}$ for $x \in E^{\times}$.

(iii) $a(\chi)$ is even.

Granting the existence of such a $\chi$, let us view $\chi$ as a character of $\operatorname{Gal}(\bar{F} / E)$ and put $\lambda=\operatorname{ind}_{E / F} \chi$. Then $\lambda$ is two-dimensional by construction, self-dual by (ii), and of trivial determinant by (i) and (1.3). As for $a(\lambda)$, the formula for the conductor of an induced representation gives

$$
a\left(\operatorname{ind}_{E / F} \chi\right)=d(E / F)+a(\chi) f(E / F)
$$

([16], p. 104, Proposition 6). But we have seen already in Example 2 that $d(E / F)$ is odd. Hence $a(\lambda)$ is odd by (iii) and (2.13).

It remains to construct $\chi$. We do so in stages. First we define $\chi$ on the subgroup $F^{\times}\left(1+\pi^{d(E / F)} \mathcal{O}_{E}\right)$ of $E^{\times}$by requiring $\chi$ to coincide with $\operatorname{sign}_{E / F}$ on $F^{\times}$and to be trivial on $1+\pi^{d(E / F)} \mathcal{O}_{E}$. These requirements are compatible because $\operatorname{sign}_{E / F}$ is trivial on $1+\pi^{d(E / F)} \mathcal{O}$. Next put $u=1+\sqrt{\pi} \cdot \pi^{d(E / F)-1}$, let $U$ be the subgroup of $E^{\times}$generated by $u$, and observe that

$$
U \cap\left(F^{\times}\left(1+\pi^{d(E / F)} \mathcal{O}_{E}\right)\right)=U^{p} \subset 1+\pi^{d(E / F)} \mathcal{O}_{E} .
$$

Hence we can extend $\chi$ to a character of $F^{\times}\left(1+\pi^{d(E / F)} \mathcal{O}_{E}\right) U$ by setting $\chi(u)=$ $e^{2 \pi i / p}$. Finally, since $\mathbb{C}^{\times}$is a divisible group, we can extend $\chi$ to a homomorphism $E^{\times} \rightarrow \mathbb{C}^{\times}$, and any such extension is continuous (because $\chi$ is trivial on $1+$ $\pi^{d(E / F)} \mathcal{O}_{E}$ ) and of finite order (because $F^{\times}\left(1+\pi^{d(E / F)} \mathcal{O}_{E}\right)$ has finite index in $E^{\times}$). Property (i) holds by construction, and (ii) follows because $\operatorname{sign}_{E / F}$ is trivial on norms from $E^{\times}$. Finally, $a(\chi) \leqslant 2 d(E / F)$ because $\chi$ is trivial on $\pi^{d(E / F)} \mathcal{O}_{E}$, but $a(\chi)>2 d(E / F)-1$ because $\chi(u) \neq 1$. Hence $a(\chi)=2 d(E / F)$, and (iii) holds.

3. Reductions. Henceforth $\rho$ denotes an essentially self-dual representation of $\mathrm{W}(\bar{F} / F)$ which is of even dimension and essentially trivial determinant. As usual, $\iota \in \operatorname{Aut}(\mathbb{C})$ and $w$ is the weight of $\rho$. Our goal in this section is to reduce the proof of Theorem 1 to the calculation of a one-dimensional "relative local root number." Only in part (b) of Proposition 8 below do we need the hypothesis that $w$ is odd: until then, $w$ can be an arbitrary integer.

The first reduction is that $\rho$ may be assumed semisimple. Indeed let $\rho_{\mathrm{ss}}$ denote the semisimplification of $\rho$. Then $\operatorname{dim} \rho_{\mathrm{ss}}=\operatorname{dim} \rho, \operatorname{det} \rho_{\mathrm{ss}}=\operatorname{det} \rho$, and

$$
\left(\rho_{\mathrm{SS}}\right)^{\vee} \cong\left(\rho^{\vee}\right)_{\mathrm{sS}} \cong\left(\rho \otimes \omega^{w}\right)_{\mathrm{sS}} \cong \rho_{\mathrm{SS}} \otimes \omega^{w},
$$

so $\rho_{\mathrm{ss}}$ is again essentially self-dual of weight $w$, even dimension, and essentially trivial determinant. Hence the following proposition shows that in proving Theorem 1 we may suppose that $\rho \cong \rho_{\text {ss }}$. 
Proposition 5. $W\left(\rho^{\iota}\right) / W(\rho)=W\left(\left(\rho_{\mathrm{ss}}\right)^{\iota}\right) / W\left(\rho_{\mathrm{ss}}\right)$.

Proof. Certainly $W\left(\rho^{\iota}\right) / W(\rho)=W\left(\left(\rho^{\iota}\right)_{\mathrm{ss}}\right) / W\left(\rho_{\mathrm{ss}}\right)$, because as a function on the Grothendieck group of virtual representations of $\mathrm{W}(\bar{F} / F)$, the root number is insensitive to semisimplification. Now substitute $\left(\rho^{\iota}\right)_{\mathrm{ss}} \cong\left(\rho_{\mathrm{ss}}\right)^{\iota}$.

A representation of $\mathrm{W}(\bar{F} / F)$ is said to be of Galois type if it factors through the finite group $\mathrm{W}(\bar{F} / F) / \mathrm{W}(\bar{F} / E) \cong \operatorname{Gal}(E / F)$ for some finite Galois extension $E$ of $F$ (cf. [17], p. 10, (2.2)).

Proposition 6. Assume that $\rho$ is semisimple.

(i) There exist representations $\lambda$ and $\vartheta$ of $\mathrm{W}(\bar{F} / F)$, with $\lambda$ of Galois type, such that $\rho$ is the direct sum of $\lambda \otimes \omega^{-w / 2}$ and $\vartheta \oplus\left(\vartheta^{\vee} \otimes \omega^{-w}\right)$.

(ii) Any $\lambda$ as in (a) is self-dual, even-dimensional, and of trivial determinant.

Proof. (i) This part of the proposition does not depend on our standing assumptions that $\operatorname{dim} \rho$ is even and $\operatorname{det} \rho$ essentially trivial, and the proof is actually easier without them. Thus we shall prove that (i) holds with $\rho$ replaced by an arbitrary semisimple essentially self-dual representation $\varphi$ of $\mathrm{W}(\bar{F} / F)$. Let $w$ be the weight of $\varphi$.

Suppose first that $\varphi$ is irreducible. Then $\varphi \cong \lambda \otimes \omega^{s}$ for some $s \in \mathbb{C}$ and $\lambda$ of Galois type (cf. [1], p. 542, (4.10) and [17], p. 10, (2.2.1)). Taking determinants, we find that

$$
\operatorname{det} \varphi=\operatorname{det} \lambda \cdot \omega^{s \operatorname{dim} \lambda} .
$$

On the other hand, we have already noted that the relation $\varphi^{\vee} \cong \varphi \otimes \omega^{w}$ gives

$$
(\operatorname{det} \varphi)^{2}=\omega^{-w \operatorname{dim} \varphi} .
$$

Since $\operatorname{dim} \varphi=\operatorname{dim} \lambda$, we deduce from (3.1) and (3.2) that

$$
(\operatorname{det} \lambda)^{2}=\omega^{-(2 s+w) \operatorname{dim} \varphi} .
$$

In particular, since $\lambda$ factors through a finite group, it follows that the character $\xi=\omega^{s+w / 2}$ is of finite order, whence $\lambda \otimes \xi$ is still of Galois type. But

$$
\varphi \cong \lambda \otimes \omega^{s} \cong(\lambda \otimes \xi) \otimes \omega^{-w / 2},
$$

so after replacing $\lambda$ by $\lambda \otimes \xi$ we have $\varphi \cong \lambda \otimes \omega^{-w / 2}$ with $\lambda$ of Galois type.

Now consider the case of an arbitrary semisimple $\varphi$. Writing $\varphi \cong \varphi_{1} \oplus \varphi_{2} \oplus \cdots \oplus \varphi_{n}$ with $\varphi_{i}$ irreducible $(1 \leqslant i \leqslant n)$, we have

$$
\varphi \otimes \omega^{w / 2} \cong\left(\varphi_{1} \otimes \omega^{w / 2}\right) \oplus\left(\varphi_{2} \otimes \omega^{w / 2}\right) \oplus \cdots \oplus\left(\varphi_{n} \otimes \omega^{w / 2}\right) .
$$

Let $l$ be the number of summands on the right-hand side that are self-dual. Since the left-hand side is self-dual by assumption, the integer $n-l$ is even, say $2 m$, and after renumbering the representations $\varphi_{i}$ we may assume that $\varphi_{i} \otimes \omega^{w / 2}$ is self-dual for $1 \leqslant i \leqslant l$ and that $\varphi_{i}^{\vee} \otimes \omega^{-w / 2} \cong \varphi_{i+m} \otimes \omega^{w / 2}$ for $l+1 \leqslant i \leqslant l+m$. Now apply the result of the previous paragraph to the representation $\varphi_{i}$ for $1 \leqslant i \leqslant l$ : we see 
that $\varphi_{i} \cong \lambda_{i} \otimes \omega^{-w / 2}$ with $\lambda_{i}$ of Galois type. Thus on setting $\lambda=\lambda_{1} \oplus \lambda_{2} \oplus \cdots \oplus \lambda_{l}$ and $\vartheta=\varphi_{l+1} \oplus \varphi_{l+2} \oplus \cdots \oplus \varphi_{l+m}$ we have

$$
\lambda \otimes \omega^{-w / 2} \cong \varphi_{1} \oplus \varphi_{2} \oplus \cdots \oplus \varphi_{l}
$$

and

$$
\vartheta \oplus\left(\vartheta^{\vee} \otimes \omega^{-w}\right) \cong \varphi_{l+1} \oplus \varphi_{l+2} \oplus \cdots \oplus \varphi_{n}
$$

Since $\varphi \cong \varphi_{1} \oplus \varphi_{2} \oplus \cdots \oplus \varphi_{n}$, the assertion follows from (3.3) and (3.4).

(ii) By (i), $\rho \otimes \omega^{w / 2} \cong \lambda \oplus\left(\vartheta \otimes \omega^{w / 2}\right) \oplus\left(\vartheta^{\vee} \otimes \omega^{-w / 2}\right)$, and we have assumed that $\rho \otimes \omega^{w / 2}$ is self-dual, even-dimensional, and of trivial determinant. By inspection these same properties hold for $\left(\vartheta \otimes \omega^{w / 2}\right) \oplus\left(\vartheta^{\vee} \otimes \omega^{-w / 2}\right)$, so they hold for $\lambda$ as well.

The proof of Theorem 1 has now been reduced to the two cases $\rho=\lambda \otimes \omega^{-w / 2}$ and $\rho=\vartheta \oplus\left(\vartheta^{\vee} \otimes \omega^{-w}\right)$. We deal with the latter case first.

Proposition 7. Suppose that $\rho \cong \vartheta \oplus\left(\vartheta^{\vee} \otimes \omega^{-w}\right)$ for some representation $\vartheta$ of $\mathrm{W}(\bar{F} / F)$. Then $W\left(\rho^{\iota}\right)=W(\rho)$.

Proof. Since $\rho^{\iota} \cong \vartheta^{\iota} \oplus\left(\left(\vartheta^{\iota}\right)^{\vee} \otimes \omega^{w}\right)$, formulas (2.4) and (2.5) give both $W(\rho)=$ $\operatorname{det} \vartheta(-1)$ and $W\left(\rho^{\iota}\right)=\operatorname{det} \vartheta(-1)^{\iota}$. But $\operatorname{det} \vartheta(-1)= \pm 1$.

Next suppose that $\rho \cong \lambda \otimes \omega^{-w / 2}$, where $\lambda$ is of Galois type and furthermore selfdual, even-dimensional, and of trivial determinant. If $\lambda$ is written as a direct sum of an orthogonal representation and a symplectic representation then the orthogonal direct summand is also even-dimensional and of trivial determinant, because for the symplectic direct summand these properties are automatic. Hence the proof of Theorem 1 reduces to the following statement:

Proposition 8. Suppose that $\rho \cong \lambda \otimes \omega^{-w / 2}$ with a representation $\lambda$ of $\mathrm{W}(\bar{F} / F)$ of Galois type. Assume that either

(a) $\lambda$ is orthogonal, even-dimensional, and of trivial determinant, or

(b) $\lambda$ is symplectic and $w$ is odd.

Then $W\left(\rho^{\iota}\right)=W(\rho)$.

We shall prove Proposition 8 by reducing it to a statement about characters. Case (a) of the proposition could also be deduced from Deligne's theorem [2] relating orthogonal root numbers to Stiefel-Whitney classes, but there would be little saving in doing so, because most of the arguments that follow are needed in case (b).

The first point to note is that

$$
\frac{W\left(\rho^{\iota}\right)}{W(\rho)}= \begin{cases}W\left(\lambda^{\iota}\right) / W(\lambda) & \text { if } \iota(\sqrt{p})=\sqrt{p} \\ (-1)^{a(\lambda) w f} W\left(\lambda^{\iota}\right) / W(\lambda) & \text { if } \iota(\sqrt{p})=-\sqrt{p} .\end{cases}
$$

Indeed (2.4) gives $W(\rho)=W(\lambda)$, and (2.2) and (2.4) together give $W\left(\rho^{\iota}\right)=W\left(\lambda^{\iota}\right)$ or $W\left(\rho^{\iota}\right)=W\left(\lambda^{\iota} \otimes \eta^{w f}\right)$ according as $\iota \mid \mathbb{Q}(\sqrt{p})$ is trivial or nontrivial. The latter alternative leads via $(2.8)$ to $W\left(\rho^{\iota}\right)=(-1)^{a(\lambda) w f} W\left(\lambda^{\iota}\right)$, and (3.5) follows.

Since $\lambda$ is of Galois type it can be viewed as a representation of the finite group $G=\operatorname{Gal}(M / F)$ for some finite Galois extension $M$ of $F$, and thus we can apply 
Serre's induction theorem ([14], p. 177, Proposition 2) in case (a) and Proposition 1 in case (b). The result is a decomposition

$$
[\lambda]=\sum_{\theta} n_{\theta}\left[\theta \oplus \theta^{\vee}\right]+\sum_{(J, \sigma)} n_{J, \sigma}\left[\operatorname{ind}_{J}^{G} \sigma\right]+\sum^{(\mathrm{a})}
$$

in the Grothendieck group of virtual representations of $G$, where in the first sum $\theta$ runs over arbitrary irreducible representations of $G$ and in the second sum $J$ denotes a subgroup of $G$ and $\sigma$ a two-dimensional irreducible monomial representation of $J$ of the appropriate type - orthogonal in case (a), symplectic in case (b). Also $n_{\theta}, n_{J, \sigma} \in \mathbb{Z}$, and the term $\sum^{(\mathrm{a})}$ is present only in case (a), when it denotes an integral linear combination of classes of the form [ind ${ }_{J}^{G} \chi$ ] with $J$ a subgroup of $G$ and $\chi$ a one-dimensional character of $J$ such that $\chi^{2}=1$.

Now fix an additive character $\psi$ of $F$. Although $\lambda$ has trivial determinant, this may no longer be true for the individual summands in the second and third sums on the right-hand side of (3.6), so after taking root numbers on both sides we write the result as follows:

$$
W(\lambda)=\prod_{\theta} W\left(\theta \oplus \theta^{\vee}\right)^{n_{\theta}} \prod_{(J, \sigma)} W\left(\operatorname{ind}_{J}^{G} \sigma, \psi\right)^{n_{J, \sigma}} \prod^{(\mathrm{a})} .
$$

Here $\prod^{(\mathrm{a})}$ denotes a product of powers of root numbers of the form $W\left(\operatorname{ind}_{J}^{G} \chi\right)$ with $J$ and $\chi$ as above. Now apply $\iota$ on both sides of (3.6), take root numbers again, and divide the result by (3.7). We find

$$
W\left(\lambda^{\iota}\right) / W(\lambda)=\prod_{(J, \sigma)}\left(W\left(\operatorname{ind}_{J}^{G} \sigma^{\iota}, \psi\right) / W\left(\operatorname{ind}_{J}^{G} \sigma, \psi\right)\right)^{n_{J, \sigma}},
$$

because the ratios of root numbers arising from the product over $\theta$ are 1 by Proposition 7 (applied with $\vartheta=\theta$ and $w=0$ ) and the ratios arising from $\prod^{(\mathrm{a})}$ are trivially 1 since $\chi^{\iota}=\chi$.

Next we focus on the individual ratios $W\left(\operatorname{ind}_{J}^{G} \sigma, \psi\right) / W\left(\operatorname{ind}_{J}^{G} \sigma, \psi\right)$ appearing on the right-hand side of (3.8). By Proposition 3, for a given pair $(J, \sigma)$ there exists a subgroup $I$ of index two in $J$ and a character $\chi$ of $I$ such that $\sigma=\operatorname{ind}_{I}^{J} \chi$ and either $\chi \circ \operatorname{trans}_{I}^{J}=1_{J}$ or $\chi \circ \operatorname{trans}_{I}^{J}=\operatorname{sign}_{J / I}$ according as we are in case (a) or case (b). Since $G=\operatorname{Gal}(M / F)$, we can write $J=\operatorname{Gal}(M / K)$ and $I=\operatorname{Gal}(M / L)$ with intermediate fields $K$ and $L$ satisfying $F \subset K \subset L \subset M$ and $[L: K]=2$. Furthermore we can view $\chi$ as a character of $L^{\times}$, and the condition on $\chi$ then becomes $\chi \mid K^{\times}=1_{K}$ in case (a) and $\chi \mid K^{\times}=\operatorname{sign}_{L / K}$ in case (b). The notational convention here is that Galois groups which appear as subscripts or superscripts can be replaced by their fixed fields, and a nested pair of Galois groups can be replaced by the corresponding extension of fixed fields. Thus the equation $\sigma=$ $\operatorname{ind}_{I}^{J} \chi$ becomes $\sigma=\operatorname{ind}_{L / K} \chi$, so that $\operatorname{ind}_{J}^{G} \sigma=\operatorname{ind}_{L / F} \chi$ by the transitivity of induction. The inductivity of the root number in degree zero ([17], pp. 11-12, (2.3.2) and pp. 14-15, (3.4.1)) gives

$$
\frac{W\left(\operatorname{ind}_{J}^{G} \sigma^{\iota}, \psi\right)}{W\left(\operatorname{ind}_{J}^{G} \sigma, \psi\right)}=\frac{W\left(\chi^{\iota}, \psi_{L}\right)}{W\left(\chi, \psi_{L}\right)},
$$


where $\psi_{X}=\psi \circ \operatorname{tr}_{X / F}$ for a finite extension $X$ of $F$. We note that $\psi_{L}=\psi_{K} \circ \operatorname{tr}_{L / K}$.

Now comes the reduction to a statement about characters. We claim that to prove Proposition 8 it suffices to prove the following statement, which no longer makes any reference to $F$ :

Proposition 9. Let $K$ be a finite extension of $\mathbb{Q}_{p}$ and $L$ a quadratic extension of $K$, and put $\psi_{L}=\psi_{K} \circ \operatorname{tr}_{L / K}$, where $\psi_{K}$ is some fixed additive character of $K$. Suppose that $\chi$ is a character of $L^{\times}$satisfying either

(a) $\chi \mid K^{\times}=1_{K}$, or

(b) $\chi \mid K^{\times}=\operatorname{sign}_{L / K}$.

Then $W\left(\chi^{\iota}, \psi_{L}\right) / W\left(\chi, \psi_{L}\right)=1$ in case (a) and

$$
\frac{W\left(\chi^{\iota}, \psi_{L}\right)}{W\left(\chi, \psi_{L}\right)}= \begin{cases}1 & \text { if } \iota(\sqrt{p})=\sqrt{p} \\ (-1)^{(d(L / K)+a(\chi) f(L / K)) f_{K}} & \text { if } \iota(\sqrt{p})=-\sqrt{p}\end{cases}
$$

in case (b).

We do not assume in Proposition 9 that $\chi$ is of finite order, but this follows both from (a) and from (b): A character of $L^{\times}$is of finite order if and only if its value on a uniformizer is a root of unity, and the latter condition is satisfied because $K^{\times} \mathcal{O}_{L}^{\times}$has finite index in $L^{\times}$(in fact index $\leqslant 2$ ). Similarly, it is automatic that $\operatorname{ind}_{L / K} \chi$ is orthogonal in case (a) and symplectic in case (b): First of all, both (a) and (b) imply that $\chi \circ N_{L / K}$ is trivial, whence $\chi\left(x^{\prime}\right)=\chi^{-1}(x)$ for $x \in L^{\times}$, where $x \mapsto x^{\prime}$ is the nontrivial element of $\operatorname{Gal}(L / K)$. Thus $\operatorname{ind}_{L / K} \chi$ is self-dual; but a two-dimensional self-dual representation $\sigma$ of a finite group is orthogonal or symplectic, and $\sigma$ is sympectic if and only if $\operatorname{det} \sigma$ is trivial. Hence (1.3) shows that $\operatorname{ind}_{L / K} \chi$ is orthogonal in case (a) and symplectic in case (b). It should be added that the hypotheses of Proposition 9 do not ensure the irreducibility of $\operatorname{ind}_{L / K} \chi$, so in that sense the proposition is slightly more general than is necessary for the application.

Before proving Proposition 9, let us verify that Proposition 8 is indeed a consequence. In case (a), $\lambda$ is orthogonal and of trivial determinant, whence Serre's theorem on conductors of orthogonal representations ([14], p. 173, Théorème 1) implies that $a(\lambda)$ is even. Thus (3.5) gives $W\left(\rho^{\iota}\right) / W(\rho)=W\left(\lambda^{\iota}\right) / W(\lambda)$ for all $\iota$, so that case (a) of Proposition 8 follows from (3.8), (3.9), and Proposition 9.

In case (b) the argument starts with (3.6). The term $\sum^{(a)}$ is now absent, whence

$$
a(\lambda) \equiv \sum_{(J, \sigma)} n_{J, \sigma} a\left(\operatorname{ind}_{J}^{G} \sigma\right) \quad(\bmod 2),
$$

because $a\left(\theta \oplus \theta^{\vee}\right)=2 a(\theta)$. Combining (3.10) with (3.5) and (3.8), we find that

$$
\frac{W\left(\rho^{\iota}\right)}{W(\rho)}= \begin{cases}\prod_{(J, \sigma)}\left(\frac{W\left(\operatorname{ind}_{J}^{G} \sigma^{\iota}, \psi\right)}{W\left(\operatorname{ind}_{J}^{G} \sigma, \psi\right)}\right)^{n_{J, \sigma}} & \text { if } \iota(\sqrt{p})=\sqrt{p} . \\ \prod_{(J, \sigma)}\left((-1)^{a\left(\operatorname{ind}_{J}^{G} \sigma\right) w f} \frac{W\left(\operatorname{ind}_{J}^{G} \sigma^{\iota}, \psi\right)}{W\left(\operatorname{ind}_{J}^{G} \sigma, \psi\right)}\right)^{n_{J, \sigma}} & \text { if } \iota(\sqrt{p})=-\sqrt{p} .\end{cases}
$$

Now fix a pair $(J, \sigma)$ on the right-hand side of (3.11), and put $\tau=\operatorname{ind}_{J}^{G} \sigma$. Also write $\sigma=\operatorname{ind}_{L / K} \chi$ as before, so that $\tau=\operatorname{ind}_{L / F} \chi$. Keeping in mind that $w$ is odd in case (b) of Proposition 8, we see that this case is a consequence of (3.11), (3.9), Proposition 9, and the following lemma. 
Lemma. $a(\tau) f \equiv(d(L / K)+a(\chi) f(L / K)) f_{K}(\bmod 2)$.

Proof. The multiplicativity of the different in towers gives

$$
d(L / F)=[L: K] d(K / F)+d(L / K) f(K / F)
$$

(cf. [16], p. 51, Proposition 8). Let us insert (3.12) into the formula for the conductor of a monomial representation, namely $a(\tau)=d(L / F)+a(\chi) f(L / F)($ cf. (2.13)). Since $[L: K]=2$, we obtain

$$
a(\tau) \equiv d(L / K) f(K / F)+a(\chi) f(L / F) \quad(\bmod 2) .
$$

The stated congruence follows on multiplying both sides of $(3.13)$ by $f\left(=f\left(F / \mathbb{Q}_{p}\right)\right)$, because the residue class degree is also multiplicative in towers.

Having verified that Proposition 9 implies Proposition 8 in both cases, let us now prove Proposition 9 in case (a). Write $L=K(\sqrt{u})$ with $u \in K^{\times}$. Since $\chi \mid K^{\times}$ and $\chi^{\iota} \mid K^{\times}$are trivial, the theorem of Fröhlich-Queyrut ([5], p. 130, Theorem 3) implies that

$$
W\left(\chi^{\iota}, \psi_{L}\right) / W\left(\chi, \psi_{L}\right)=\chi^{\iota}(\sqrt{u}) / \chi(\sqrt{u})
$$

The triviality of $\chi \mid K^{\times}$not only renders the choice of square root in $\chi(\sqrt{u})$ irrelevant but also shows that $\chi(\sqrt{u})= \pm 1$, whence the right-hand side of (3.14) is 1 , as required.

The proof of Proposition 9 in case (b) involves a further division into cases according as $L / K$ is ramified or unramified. Suppose first that $L / K$ is unramified. Then $d(L / K)=0$ and $f(L / K)=2$, so the statement to be proved is that $W\left(\chi^{\iota}, \psi_{L}\right)=W\left(\chi, \psi_{L}\right)$ for all $\iota$. Let $\eta_{L}$ denote the unramified quadratic character of $L^{\times}$, and put $\zeta=\chi \cdot \eta_{L}$. Since $L / K$ is unramified, a uniformizer of $K$ is also a uniformizer of $L$, and therefore $\eta_{L} \mid K^{\times}=\operatorname{sign}_{L / K}$. Hence the condition $\chi \mid K^{\times}=\operatorname{sign}_{L / K}$ implies that $\zeta \mid K^{\times}$is trivial, whence the result of Fröhlich-Queyrut gives $W\left(\zeta, \psi_{L}\right)=\zeta(\sqrt{u})$ with $u \in K^{\times}$and $L=K(\sqrt{u})$. Note once again that the choice of square root is irrelevant. We also have $W\left(\zeta^{\iota}, \psi_{L}\right)=\zeta^{\iota}(\sqrt{u})$, and since $\zeta(\sqrt{u})= \pm 1$ we deduce that $W\left(\zeta^{\iota}, \psi_{L}\right)=W\left(\zeta, \psi_{L}\right)$. But $\zeta$ is the unramified quadratic twist of $\chi$, so

$$
\frac{W\left(\zeta^{\iota}, \psi_{L}\right)}{W\left(\zeta, \psi_{L}\right)}=\frac{(-1)^{a\left(\chi^{\iota}\right)+n\left(\psi_{L}\right)} W\left(\chi^{\iota}, \psi_{L}\right)}{(-1)^{a(\chi)+n\left(\psi_{L}\right)} W\left(\chi, \psi_{L}\right)}=\frac{W\left(\chi^{\iota}, \psi_{L}\right)}{W\left(\chi, \psi_{L}\right)}
$$

by (2.8). As $W\left(\zeta^{\iota}, \psi_{L}\right) / W\left(\zeta, \psi_{L}\right)=1$ we have $W\left(\chi^{\iota}, \psi_{L}\right) / W\left(\chi, \psi_{L}\right)=1$ also.

Next suppose that $L / K$ is ramified. Then $f(L / K)=1$. On the other hand, $\iota\left(\sqrt{q_{K}}\right)=-\sqrt{q_{K}}$ if and only if $\iota(\sqrt{p})=-\sqrt{p}$ and $f_{K}$ is odd, so the statement to be proved is equivalent to

$$
\frac{W\left(\chi^{\iota}, \psi_{L}\right)}{W\left(\chi, \psi_{L}\right)}= \begin{cases}1 & \text { if } \iota\left(\sqrt{q_{K}}\right)=\sqrt{q_{K}} . \\ (-1)^{a(\chi)+d(L / K)} & \text { if } \iota\left(\sqrt{q_{K}}\right)=-\sqrt{q_{K}} .\end{cases}
$$

We can reformulate (3.15) using the "relative local root number"

$$
W_{L / K}(\chi)=W\left(\chi, \psi_{L}\right) / W\left(\operatorname{sign}_{L / K}, \psi_{K}\right) .
$$

Note first of all that the right-hand side of (3.16) is independent of the choice of $\psi_{K}$ by virtue of (2.7) and our assumption that $\psi_{L}=\psi_{K} \circ \operatorname{tr}_{L / K}$ and $\chi \mid K^{\times}=\operatorname{sign}_{L / K}$. Furthermore (3.15) becomes

$$
\frac{W_{L / K}\left(\chi^{\iota}\right)}{W_{L / K}(\chi)}= \begin{cases}1 & \text { if } \iota\left(\sqrt{q_{K}}\right)=\sqrt{q_{K}} . \\ (-1)^{a(\chi)-d(L / K)} & \text { if } \iota\left(\sqrt{q_{K}}\right)=-\sqrt{q_{K}} .\end{cases}
$$

We shall prove (3.17) in the next section. The following remark will be helpful: 
Proposition 10. $W_{L / K}(\chi)= \pm 1$.

Proof. We have $W\left(\operatorname{ind}_{L / K} \chi^{-1}\right) / W\left(\operatorname{ind}_{L / K} \chi\right)=W\left(\chi^{-1}, \psi_{L}\right) / W\left(\chi, \psi_{L}\right)$ by the inductivity of the root number in degree zero. But $\operatorname{ind}_{L / K} \chi^{-1} \cong \operatorname{ind}_{L / K} \chi$, so it follows that $W\left(\chi^{-1}, \psi_{L}\right)=W\left(\chi, \psi_{L}\right)$. Hence $(2.5)$ gives $W\left(\chi, \psi_{L}\right)^{2}=\chi(-1)$ as well as $W\left(\operatorname{sign}_{L / K}, \psi_{K}\right)^{2}=\operatorname{sign}_{L / K}(-1)$. Thus

$$
W_{L / K}(\chi)^{2}=\frac{W\left(\chi, \psi_{L}\right)^{2}}{W\left(\operatorname{sign}_{L / K}, \psi_{K}\right)^{2}}=\frac{\chi(-1)}{\operatorname{sign}_{L / K}(-1)},
$$

which is 1 because $\chi \mid K^{\times}=\operatorname{sign}_{L / K}$.

4. The relative local root number. To recapitulate, $K$ is a finite extension of $\mathbb{Q}_{p}, L$ is a ramified quadratic extension of $K$, and $\chi$ is a character of $L^{\times}$satisfying the condition $\chi \mid K^{\times}=\operatorname{sign}_{L / K}$. We fix an additive character $\psi_{K}$ of $K$ and set $\psi_{L}=\psi_{K} \circ \operatorname{tr}_{L / K}$, and we define $W_{L / K}(\chi)$ by (3.16). Our goal is to prove (3.17).

Let $Z$ denote the group $\mathcal{O}_{L}^{\times} / \mathcal{O}_{K}^{\times}$, the quotient of $\mathcal{O}_{L}^{\times}$by its closed subgroup $\mathcal{O}_{K}^{\times}$. Given $\gamma \in L^{\times}$, we define a subset $Z_{\gamma}$ of $Z$ by

$$
Z_{\gamma}=\left\{z \in Z: \operatorname{tr}_{L / K}(y / \gamma) \in \mathcal{O}_{K}^{\times} \text {for some (and hence for all) } y \in z\right\},
$$

and we consider the $\mathbb{C}^{\times}$-valued function on $Z_{\gamma}$ given by

$$
z \mapsto \chi^{-1}(y / \gamma) \operatorname{sign}_{L / K}\left(\operatorname{tr}_{L / K}(y / \gamma)\right)
$$

where $y \in z$. That (4.1) is well-defined follows from our assumption $\chi \mid K^{\times}=$ $\operatorname{sign}_{L / K}$. We would like to choose $\gamma$ so that (4.1) factors through the image of $Z_{\gamma}$ in a finite quotient of $Z$.

For a positive integer $n$ let $Z(n)$ denote the image in $Z$ of the subgroup $1+\mathfrak{p}_{L}^{n}$ of $\mathcal{O}_{L}^{\times}$. In addition, put $Z(0)=Z$. Then $Z / Z(n)$ is finite in all cases. Writing $v_{K}$ and $v_{L}$ for the valuations on $K$ and $L$, and choosing $\gamma$ so that

$$
v_{L}(\gamma)=a(\chi)-d(L / K)
$$

we claim that $Z_{\gamma}$ is a union of cosets of $Z(a(\chi))$ and that the value of (4.1) at $z \in Z_{\gamma}$ depends only on the coset $z Z(a(\chi))$.

To verify the claim, let $y \in \mathcal{O}_{L}^{\times}$represent an element of $Z_{\gamma}$, and take $x \in \mathcal{O}_{L}$. Also let $\pi_{K}$ and $\pi_{L}$ denote uniformizers of $\mathcal{O}_{K}$ and $\mathcal{O}_{L}$. Then

$$
y\left(1+\pi_{L}^{a(\chi)} x\right) / \gamma=y / \gamma+\pi_{K}^{d(L / K)}\left(x^{\prime} / \pi_{L}^{d(L / K)}\right)
$$

with $x^{\prime} \in \mathcal{O}_{L}$. But $\pi_{L}^{d(L / K)}$ generates the different ideal of $L / K$, so we obtain

$$
\operatorname{tr}_{L / K}\left(y\left(1+\pi_{L}^{a(\chi)} x\right) / \gamma\right)=\operatorname{tr}_{L / K}(y / \gamma)+\pi_{K}^{d(L / K)} x^{\prime \prime}
$$

with $x^{\prime \prime} \in \mathcal{O}_{K}$. Hence $Z_{\gamma}$ is indeed a union of cosets of $Z(a(\chi))$. The second part of our claim also follows, because $\pi_{K}^{d(L / K)}$ generates the conductor of $\operatorname{sign}_{L / K}$.

In the following proposition, we assume that $\gamma$ is chosen as in (4.2). 
Proposition 11. Let $Y$ be a set of representatives in $\mathcal{O}_{L}^{\times}$for the distinct cosets of $Z(a(\chi))$ in $Z_{\gamma}$. Then

$$
W_{L / K}(\chi)=c \sum_{y \in Y} \chi^{-1}(y / \gamma) \operatorname{sign}_{L / K}\left(\operatorname{tr}_{L / K}(y / \gamma)\right)
$$

with $c=q_{K}^{(a(\chi)-d(L / K)) / 2-[a(\chi) / 2]}$.

Formula (3.17) follows at once from the proposition. Indeed let us apply $\iota$ to both sides of the formula for $W_{L / K}(\chi)$. Since $W_{L / K}(\chi)= \pm 1$ (Proposition 10), the left-hand side is unchanged, but on the right-hand side $\chi$ is replaced by $\chi^{\iota}$ and $c$ is either unchanged or multiplied by $(-1)^{a(\chi)-d(L / K)}$ according as $\iota\left(\sqrt{q_{K}}\right)$ is $\sqrt{q_{K}}$ or $-\sqrt{q_{K}}$.

We turn now to the proof of Proposition 11. In the following lemma $n$ denotes a nonnegative integer.

Lemma. $|Z / Z(n)|=q_{K}^{[n / 2]}$.

Proof. We claim that the map $\mathcal{O}_{K} / \mathfrak{p}_{K}^{[n / 2]} \rightarrow Z / Z(n)$ given for $x \in \mathcal{O}_{K}$ by

$$
x+\mathfrak{p}_{K}^{[n / 2]} \mapsto\left(1+\pi_{L} x\right) \mathcal{O}_{K}^{\times} Z(n)
$$

is a well-defined bijection. To see this, first note that $\mathcal{O}_{L}=\mathcal{O}_{K} \oplus \pi_{L} \mathcal{O}_{K}$, whence $\mathcal{O}_{L}^{\times}=\mathcal{O}_{K}^{\times}+\pi_{L} \mathcal{O}_{K}$ as sets. Thus every element of $Z$ has a unique representative of the form $1+\pi_{L} x$ with $x \in \mathcal{O}_{K}$. Consequently it suffices to check that two such representatives $1+\pi_{L} x$ and $1+\pi_{L} x^{\prime}$ determine the same class in $Z / Z(n)$ if and only if $x \equiv x^{\prime}$ modulo $\mathfrak{p}_{K}^{[n / 2]}$. This condition is certainly sufficient, for it implies that $x \equiv x^{\prime}$ modulo $\mathfrak{p}_{L}^{n-1}$. To verify that the congruence is necessary, take $x \neq x^{\prime}$ and observe that

$$
v_{L}\left(-1+\left(1+\pi_{L} x\right) /\left(1+\pi_{L} x^{\prime}\right)\right)=1+2 v_{K}\left(x-x^{\prime}\right) .
$$

In particular, the left-hand side of (4.3) is odd. Hence $\left(1+\pi_{L} x\right) /\left(1+\pi_{L} x^{\prime}\right)=$ $u\left(1+\pi_{L}^{n} y\right)$ with $u \in \mathcal{O}_{K}^{\times}$and $y \in \mathcal{O}_{L}$ only if $v_{K}(u-1) \geqslant n / 2$. By (4.3) this last condition implies that $x \equiv x^{\prime} \bmod \mathfrak{p}_{K}^{[n / 2]}$.

Proof of Proposition 11. Let $d z$ be the Haar measure on $Z$ of total mass 1. Applying the lemma, we see that the proposition is equivalent to the formula

$$
W_{L / K}(\chi)=q_{K}^{(a(\chi)-d(L / K)) / 2} \int_{Z_{\gamma}} \chi^{-1}(z / \gamma) \operatorname{sign}_{L / K}\left(\operatorname{tr}_{L / K}(z / \gamma)\right) d z .
$$

For the sake of completeness we shall derive (4.4) from scratch, but it is merely a slight generalization of Proposition 7 of [9] (where $a(\chi)-d(L / K)$ is assumed even) and Proposition 1 of [11] (where $p$ is assumed odd).

By definition, $n\left(\psi_{L}\right)$ is the largest integer $n$ such that $\psi_{L}$ is trivial on $\mathfrak{p}_{L}^{-n}$. But $\psi_{L}=\psi_{K} \circ \operatorname{tr}_{L / K}$ and $\operatorname{tr}_{L / K}\left(\mathfrak{p}_{L}^{-n}\right)=\mathfrak{p}_{K}^{-m}$ for some $m$ depending on $n$, so $\psi_{L} \mid \mathfrak{p}_{L}^{-n}$ is trivial if and only if $\operatorname{tr}_{L / K}\left(\mathfrak{p}_{L}^{-n}\right) \subset \mathfrak{p}_{K}^{-n\left(\psi_{K}\right)}$. The latter property holds if and only if $\mathfrak{p}_{L}^{n-2 n\left(\psi_{K}\right)}$ contains the different ideal of $L / K$, so we conclude that

$$
n\left(\psi_{L}\right)=d(L / K)+2 n\left(\psi_{K}\right) .
$$


Now choose $\beta \in K^{\times}$so that

$$
v_{K}(\beta)=d(L / K)+n\left(\psi_{K}\right),
$$

and put $\alpha=\beta \gamma$. Using (4.2), (4.5), and (4.6), we obtain

$$
v_{L}(\alpha)=a(\chi)+n\left(\psi_{L}\right) .
$$

In connection with (4.6) we recall that $d(L / K)=a\left(\operatorname{sign}_{L / K}\right)$.

Let $d_{K} x$ and $d_{L} y$ be the self-dual Haar measures on $K$ and $L$ relative to $\psi_{K}$ and $\psi_{L}$ respectively. In view of (4.6) and (4.7), the standard formula for the epsilon factor of a ramified character gives

$$
W\left(\operatorname{sign}_{L / K}, \psi_{K}\right)=q_{K}^{\left(d(L / K)+n\left(\psi_{K}\right)\right) / 2} \int_{\mathcal{O}_{K}^{\times}} \operatorname{sign}_{L / K}(x / \beta) \psi_{K}(x / \beta) d_{K} x
$$

and

$$
W\left(\chi, \psi_{L}\right)=q_{L}^{\left(a(\chi)+n\left(\psi_{L}\right)\right) / 2} \int_{\mathcal{O}_{L}^{\times}} \chi^{-1}(y / \alpha) \psi_{L}(y / \alpha) d_{L} y
$$

(cf. [17], p. 14, (3.2.6.2) and p. 15, (3.4.7)). Here we are using the fact that $\operatorname{sign}_{L / K}$ and $\chi$ are of finite order, hence unitary.

Next we compare measures. The restrictions of $d_{K} x$ to $\mathcal{O}_{K}^{\times}$and $d_{L} y$ to $\mathcal{O}_{L}^{\times}$are Haar measures on these groups, so the quotient measure $d_{L} y / d_{K} x$ on $Z$ coincides up to a scalar factor with $d z$. To compute the scalar, recall that the self-dual measure $d_{K} x$ on $K$ gives $\mathcal{O}_{K}$ measure $q_{K}^{-n\left(\psi_{K}\right) / 2}$ and hence $\mathcal{O}_{K}^{\times}$measure $\left(1-1 / q_{K}\right) q_{K}^{-n\left(\psi_{K}\right) / 2}$. Similarly $d_{L} y$ gives $\mathcal{O}_{L}^{\times}$measure $\left(1-1 / q_{L}\right) q_{L}^{-n\left(\psi_{L}\right) / 2}$. But $q_{K}=q_{L}$, so $d_{L} y / d_{K} x$ gives $Z$ measure $q_{K}^{\left(-n\left(\psi_{L}\right)+n\left(\psi_{K}\right)\right) / 2}$. It follows that

$$
d_{L} y=q_{K}^{\left(-n\left(\psi_{L}\right)+n\left(\psi_{K}\right)\right) / 2} d_{K} x d z,
$$

because $d z$ gives $Z$ measure 1 .

Given $z \in Z$, let $\tilde{z} \in \mathcal{O}_{L}^{\times}$denote a representative of $z$, and observe that if $y=x \tilde{z}$ with $x \in \mathcal{O}_{K}^{\times}$then

$$
\chi^{-1}(y)=\chi^{-1}(\tilde{z}) \operatorname{sign}_{L / K}(x)
$$

and

$$
\psi_{L}(y / \alpha)=\psi_{K}\left(x \operatorname{tr}_{L / K}(\tilde{z} / \gamma) / \beta\right) .
$$

Inserting (4.10), (4.11), and (4.12) on the right-hand side of (4.9), and setting $u=\operatorname{tr}_{L / K}(\tilde{z} / \gamma)$, we obtain

$$
W\left(\chi, \psi_{L}\right)=q_{K}^{\left(a(\chi)+n\left(\psi_{K}\right)\right) / 2} \int_{Z} \chi^{-1}(\tilde{z} / \alpha)\left(\int_{\mathcal{O}_{K}^{\times}} \operatorname{sign}_{L / K}(x) \psi_{K}(x u / \beta) d_{K} x\right) d z .
$$

But the inner integral in (4.13) is 0 unless $u \in \mathcal{O}_{K}^{\times}$, so we can restrict the $z$ integration to $Z_{\gamma}$ and replace $x$ by $x / u$ in the inner integral (recall that $d_{K} x$ is a Haar measure on $\left.\mathcal{O}_{K}^{\times}\right)$. Observing that $\chi^{-1}(\tilde{z} / \alpha)=\chi^{-1}(\tilde{z} / \gamma) \operatorname{sign}_{L / K}(1 / \beta)$ and then referring to (4.8), we obtain (4.4). 
5. Generalization: representations of the Weil-Deligne group. We return to our finite extension $F$ of $\mathbb{Q}_{p}$ and to the associated notation: $\mathcal{O}$ for the ring of integers of $F, \mathfrak{p}$ for the maximal ideal of $\mathcal{O}, q$ for the order of the residue class field $\mathcal{O} / \mathfrak{p}$, and so on. We would like to generalize Theorem 1 to representations of the Weil-Deligne group $\mathrm{WD}(\bar{F} / F)$. For our purposes, a representation of $\mathrm{WD}(\bar{F} / F)$ is an ordered pair $\rho=(\rho, N)$, where $\rho$ is a representation of $\mathrm{W}(\bar{F} / F)$ and $N$ a nilpotent endomorphism of the space of $\rho$ such that

$$
\rho(g) N \rho(g)^{-1}=\omega(g) N \quad(g \in \mathrm{W}(\bar{F} / F)),
$$

(cf. [1], p. 568, (8.4.1) and [17], p. 20, (4.1.2)). We view $\rho$ itself as a representation of $\operatorname{WD}(\bar{F} / F)$ via the identification $\rho=(\rho, 0)$. Given an additive character $\psi$ of $F$ and an arbitrary representation $\boldsymbol{\rho}=(\rho, N)$ of $\mathrm{WD}(\bar{F} / F)$, we set

$$
W(\boldsymbol{\rho}, \psi)=W(\rho, \psi) \Delta(\boldsymbol{\rho})
$$

where $\Delta(\boldsymbol{\rho})$ is defined as follows.

Let $I$ denote the inertia subgroup of $\mathrm{W}(\bar{F} / F)$ and $V$ the space of $\rho$, and write $V^{I}$ for the subspace of $v \in V$ such that $\rho(i) v=v$ for all $i \in I$. Also put $V_{N}=\operatorname{ker} N$ and $V_{N}^{I}=V_{N} \cap V^{I}$, and let $\Phi \in \mathrm{W}(\bar{F} / F)$ denote any element which acts as the inverse of the Frobenius automorphism on the residue class field of $\bar{F}$. Then $V^{I}$ is stable under $\rho(\Phi)$, and the restriction of $\rho(\Phi)$ to $V^{I}$ is independent of the choice of $\Phi$. In fact (5.1) shows that $V_{N}$ is stable under $\rho(\Phi)$ as well, so $\rho(\Phi)$ acts on the quotient space $V^{I} / V_{N}^{I}$, and the resulting automorphism $\rho(\Phi) \mid V^{I} / V_{N}^{I}$ is independent of the choice of $\Phi$. We put

$$
\Delta(\boldsymbol{\rho})=\frac{\delta(\boldsymbol{\rho})}{|\delta(\boldsymbol{\rho})|}
$$

with

$$
\delta(\boldsymbol{\rho})=\operatorname{det}\left(-\rho(\Phi) \mid V^{I} / V_{N}^{I}\right)
$$

(cf. [17], pp. 20-21, (4.1.6)). If $N=0$ then $V_{N}^{I}=V^{I}$ and hence $\Delta=1$, so the value of $W(\rho, \psi)$ when $\rho$ is viewed as a representation of $\mathrm{W}(\bar{F} / F)$ is the same as the value when $\rho$ is identified with the representation $(\rho, 0)$ of $\mathrm{WD}(\bar{F} / F)$.

To formulate an analogue of Theorem 1, we need an action of Aut($(\mathbb{C})$ on isomorphism classes of representations of $\mathrm{WD}(\bar{F} / F)$ and also a notion of essential self-duality. Let us start with the action of $\operatorname{Aut}(\mathbb{C})$. This is a straightforward extension of the action already defined on isomorphism classes of representations of $\mathrm{W}(\bar{F} / F)$ : Given $\boldsymbol{\rho}=(\rho, N)$ and $\iota \in \operatorname{Aut}(\mathbb{C})$, choose a basis for the space of $\rho$, so that $\rho$ can be viewed as a matrix representation $\rho: \mathrm{W}_{F} \rightarrow \operatorname{GL}(n, \mathbb{C})$ and $N$ as an $n \times n$ nilpotent matrix over $\mathbb{C}$. We set $\rho^{\iota}=\left(\rho^{\iota}, N^{\iota}\right)$, where $\rho^{\iota}$ is as previously defined and $N^{\iota}$ is likewise the matrix obtained by applying $\iota$ to the coefficients of $N$. As before, the isomorphism class of $\boldsymbol{\rho}^{\iota}$ is independent of the basis chosen.

The dual of $\boldsymbol{\rho}$ is $\boldsymbol{\rho}^{\vee}=\left(\rho^{\vee},-N^{\mathrm{t}}\right)$, where $N^{\mathrm{t}}$ is the transpose of $N$. We call $\boldsymbol{\rho}$ self-dual if $\boldsymbol{\rho}^{\vee} \cong \boldsymbol{\rho}$ and essentially self-dual of weight $w$ if $\boldsymbol{\rho} \otimes \omega^{w / 2}$ is self-dual. Here we should recall that the tensor product of $\boldsymbol{\rho}_{1}=\left(\rho_{1}, N_{1}\right)$ and $\boldsymbol{\rho}_{2}=\left(\rho_{2}, N_{2}\right)$ is the representation

$$
\boldsymbol{\rho}_{1} \otimes \boldsymbol{\rho}_{2}=\left(\rho_{1} \otimes \rho_{2}, N_{1} \otimes \mathrm{id}_{2}+\mathrm{id}_{1} \otimes N_{2}\right)
$$


where $\operatorname{id}_{j}(j=1,2)$ is the identity automorphism of the space of $\rho_{j}$. In particular, tensoring with $\omega^{w / 2}=\left(\omega^{w / 2}, 0\right)$ gives $\boldsymbol{\rho} \otimes \omega^{w / 2}=\left(\rho \otimes \omega^{w / 2}, N\right)$, so we see that if $\boldsymbol{\rho}$ is essentially self-dual of weight $w$ then so is $\rho$. In this situation we will also say that the determinant of $\boldsymbol{\rho}$ is essentially trivial if the determinant of $\rho$ is. Thus $\operatorname{det} \rho$ is essentially trivial if $\operatorname{det}\left(\rho \otimes \omega^{w / 2}\right)$ is trivial.

Proposition 12. Let $\boldsymbol{\rho}$ be a representation of $\mathrm{WD}(\bar{F} / F)$.

(i) If $\boldsymbol{\rho}$ is essentially self-dual and $\operatorname{det} \boldsymbol{\rho}$ is essentially trivial then $W(\boldsymbol{\rho}, \psi)$ is equal to \pm 1 and independent of $\psi$.

(ii) If $\boldsymbol{\rho}$ is essentially self-dual then so is $\boldsymbol{\rho}^{\iota}$.

(iii) Suppose that $\boldsymbol{\rho}$ is essentially self-dual and $\operatorname{dim} \boldsymbol{\rho}$ is even. If $\operatorname{det} \boldsymbol{\rho}$ is essentially trivial then so is $\operatorname{det} \boldsymbol{\rho}^{\iota}$.

(iv) If $\boldsymbol{\rho}$ is essentially self-dual, even-dimensional, and of essentially trivial determinant then $W\left(\boldsymbol{\rho}^{\iota}, \psi\right)$ is equal to \pm 1 and independent of $\psi$.

Proof. (i) That $W(\boldsymbol{\rho}, \psi)$ is independent of $\psi$ follows from (5.2) and part (i) of Proposition 4. The same citations reduce the proof of the equality $W(\boldsymbol{\rho}, \psi)= \pm 1$ to a proof that $\Delta(\boldsymbol{\rho})= \pm 1$. Put $b(\boldsymbol{\rho})=\operatorname{dim} V^{I} / V_{N}^{I}$. Then

$$
\delta(\boldsymbol{\rho}) \delta\left(\boldsymbol{\rho}^{\vee}\right)=q^{b(\boldsymbol{\rho})}
$$

(cf. [12], p. 144, part (ii) of the lemma). Since $\boldsymbol{\rho}^{\vee} \cong \boldsymbol{\rho} \otimes \omega^{w}$ and $\left(\rho \otimes \omega^{w}\right)(\Phi)=$ $q^{-w} \rho(\Phi)$, it follows from (5.4) and (5.5) that

$$
\delta(\boldsymbol{\rho})^{2}=q^{b(\boldsymbol{\rho})(w+1)} .
$$

In particular, $|\delta(\boldsymbol{\rho})|^{2}=\delta(\boldsymbol{\rho})^{2}$ and therefore $\Delta(\boldsymbol{\rho})^{2}=1$ by (5.3). We remark that in the essentially symplectic case the fact that $W(\boldsymbol{\rho}, \psi)= \pm 1$ was proved by Gross ([8], p. 533, Proposition 3.14).

(ii) This is a straightforward verification similar to the proof of part (ii) of Proposition 4 , but with $\rho$ now replaced by $(\rho, N)$.

(iii) Since the essential triviality of $\operatorname{det} \rho$ is purely a statement about $\operatorname{det} \rho$, this follows from part (iii) of Proposition 4.

(iv) This follows from (i), (ii), and (iii).

Henceforth, if $\boldsymbol{\rho}$ is essentially self-dual and $\operatorname{det} \boldsymbol{\rho}$ essentially trivial then we write $W(\boldsymbol{\rho}, \psi)$ simply as $W(\boldsymbol{\rho})$.

Theorem 2. Suppose that $\boldsymbol{\rho}$ is essentially self-dual of odd weight, even dimension, and essentially trivial determinant. Then $W\left(\boldsymbol{\rho}^{\iota}\right)=W(\boldsymbol{\rho})$.

Proof. It follows from (5.6) that $|\delta(\boldsymbol{\rho})|=q^{b(\boldsymbol{\rho})(w+1) / 2}$, whence (5.4) becomes

$$
\Delta(\boldsymbol{\rho})=\frac{1}{q^{b(\boldsymbol{\rho})(w+1) / 2}} \operatorname{det}\left(-\rho(\Phi) \mid V^{I} / V_{N}^{I}\right) .
$$

Furthermore $w$ is odd, so the exponent of $q$ on the right-hand side of (5.7) is an integer. Now consider the determinant on the right-hand side. The dependence of $V^{I}$ and $V_{N}^{I}$ on $\boldsymbol{\rho}$ is not quite explicit in the notation, but on recalling the definitions we see that $V^{I}$ and $V_{N}^{I}$ are unchanged - as are therefore $V^{I} / V_{N}^{I}$ and $b(\boldsymbol{\rho})$ - when $\boldsymbol{\rho}$ is replaced by $\boldsymbol{\rho}^{\iota}$. Hence the effect of applying $\iota$ to the right-hand of (5.7) is to replace $\rho(\Phi)$ by $\rho^{\iota}(\Phi)$, but $\iota$ leaves the left-hand side of (5.7) unchanged, because $\Delta(\boldsymbol{\rho})= \pm 1$. Hence $\Delta\left(\boldsymbol{\rho}^{\iota}\right)=\Delta(\boldsymbol{\rho})$. The theorem now follows from (5.2) and Theorem 1. 


\section{REFERENCES}

1. P. Deligne, Les constantes des équations fonctionelles des fonctions L, Modular Functions of One Variable, II, Lect. Notes in Math. 349, Springer-Verlag, 1973, pp. 501-595.

2. P. Deligne, Les constantes locales de l'équation fonctionelle de la fonction L d'Artin d'une représentation orthogonale, Invent. Math. 35 (1976), 299 - 316.

3. P. Deligne, Valeurs de fonctions L et périodes d'intégrales, Automorphic Forms, Representations, and L-Functions, Proc. Symp. Pure Math. Vol. 33 - Part 2, Amer. Math. Society, Providence, 1979, pp. $313-346$.

4. W. Feit, Characters of Finite Groups, W. A. Benjamin, New York, 1967.

5. A. Fröhlich and J. Queyrut, On the functional equation for the Artin L-function for characters of real representations, Invent. Math. 20 (1973), 125-138.

6. P. X. Gallagher, Determinants of representations of finite groups, Abh. Math. Sem. Univ. Hamburg 28 (1965), 162-167.

7. D. M. Goldschmidt and I. M. Isaacs, Schur indices in finite groups, J. Algebra 33 (1975), 191 $-199$.

8. B. H. Gross, L-functions at the central critical point, Motives, Proc. Symp. Pure Math. Vol. 55 - Part 1, Amer. Math. Society, Providence, 1994, pp. 527 - 535.

9. D. E. Rohrlich, Root numbers of Hecke L-functions of CM fields, Amer. J. Math. 104 (1982), $517-543$.

10. D. E. Rohrlich, The vanishing of certain Rankin-Selberg convolutions, Automorphic Forms and Analytic Number Theory, Les publications CRM, Montreal, 1990, pp. 123 - 133.

11. D. E. Rohrlich, Root numbers of Jacobi-sum Hecke characters, Illinois J. Math. 36 (1992), $155-176$.

12. D. E. Rohrlich, Elliptic curves and the Weil-Deligne group, Elliptic Curves and Related Topics, CRM Proceedings \& Lecture Notes Vol. 4, Amer. Math. Soc., Providence, 1994, pp. 125 $-157$.

13. J-P. Serre, Sur la rationalité des représentations d'Artin, Ann. of Math. 72 (1960), 405 - 420.

14. J-P. Serre, Conducteurs d'Artin des caractères réels, Invent. Math. 14 (1971), 173-183.

15. J-P. Serre, Linear Representations of Finite Groups, translated from the French by L. S. Scott, Springer-Verlag, 1977.

16. J-P. Serre, Local Fields, translated from the French by M. J. Greenberg, Springer-Verlag, 1979.

17. J. Tate, Number theoretic background, Automorphic Forms, Representations, and L-Functions, Proc. Symp. Pure Math. Vol. 33 - Part 2, Amer. Math. Society, Providence, 1979, pp. 3 - 26.

Department of Mathematics and Statistics, Boston University, Boston, MA 02215 USA

E-mail address: rohrlich@math.bu.edu 